\title{
Thermally induced rarefied gas flow in a three-dimensional enclosure with square cross-section
}

\author{
Lianhua Zhu, ${ }^{1}$ Xiaofan Yang, ${ }^{2}$ and Zhaoli Guo ${ }^{1, *}$ \\ ${ }^{1}$ State Key Laboratory of Coal Combustion, School of Power and Energy Engineering, \\ Huazhong University of Science and Technology, Wuhan, 430074, China \\ ${ }^{2}$ Faculty of Geographical Science, Beijing Normal University, Beijing, 100875, China
}

\begin{abstract}
Rarefied gas flow in a three-dimensional enclosure induced by nonuniform temperature distribution is numerically investigated. The enclosure has a square channel-like geometry with alternatively heated closed ends and lateral walls with a linear temperature distribution. A recently proposed implicit discrete velocity method with a memory reduction technique is used to numerically simulate the problem based on the nonlinear Shakhov kinetic equation. The Knudsen number dependencies of the vortexes pattern, slip velocity at the planar walls and edges, and heat transfer are investigated. The influences of the temperature ratio imposed at the ends of the enclosure and the geometric aspect ratio are also evaluated. The overall flow pattern shows similarities with those observed in two-dimensional configurations in literature. However, new features due to the three-dimensionality are observed with new types of vortexes that are not identified in previous studies on similar twodimensional enclosures at high Knudsen and small aspect ratios.
\end{abstract}

\section{INTRODUCTION}

Thermal transpiration (also known as thermal creep flow) is a rarefied gas flow phenomenon occurs near a nonisothermal solid boundary and is solely driven the tangential heat flux along the wall surface [1]. This non-equilibrium flow phenomenon has inspired numerous studies ever since its discovery [2] and the first theoretical investigation [3] in the late 1800s. Partially due to its application as a Knudsen pump [4-6], the thermal transpiration in channel- or pipelike geometries has been studied extensively both by experiments [7-9] and numerical simulations [10-14] in the past few decades. In addition to the scientific importance and popularity in evaluating novel numerical tools, the thermal creep flow in an enclosure has a strong application background in vacuum packaged and micro electro-mechanical systems (MEMS) industries [15, 16] and crystal growth experiments [17].

The thermal transpiration in enclosures, however, receives growing interest only recently. Unlike the cases in simple channels with long aspect ratios and small temperature differences, as assumed in the above works, the thermal transpiration in an enclosure has much more complex features and is computationally more challenging to simulate. The extra geometrical confinements introduce inhomogeneous temperature variation in the bulk region and accordingly, other types of thermally induced flow such as thermal stress flow [1] are triggered and interplay with the thermal transpiration near the boundary. Such complex features have led to a variety of investigations. For example, in Refs. [18] and [19], for the rarefied flow in a two-dimensional cavity with nonuniform wall temperatures, it is observed that gas near the non-isothermal wall is not necessarily driven from cold to hot, which is contradictory to the predictions by the thermal transpiration theory $[1,3]$. The odds inverted thermal creep flow can be explained by the relative contribution of the shear stress and tangential heat flux along the wall according to Refs. [18, 20]. In Ref. [20], an approach that decomposes the solutions into ballistic and collision parts is introduced to interpret the thermal creep flow patterns in a two-dimensional cavity. In Ref. [21], the full Boltzmann equation solver reveals that the velocity field of thermally induced flow is clearly affected by the molecular interaction model.

Due to its non-equilibrium nature, the mathematical description of thermally induced rarefied flow is mainly based upon the Boltzmann or the kinetic model equations, as the classical continuum models such as the Euler and NavierStokes equations are only applicable in the slip flow regime, i.e., slightly rarefied flows [22]. However, the kinetic equations are computationally much more expensive to solve. The extremely low-speed character of such flows makes it even more difficult for applying direct simulation Monte Carlo (DSMC) method [23] which is a common tool for rarefied gas flows. An early attempt for such flow in an enclosure has been reported in Ref. [17], where the thermally induced flow in a two-dimensional microcavity has been simulated with the DSMC method which took 5 days of CPU time for a single simulation [17]. The enormous computational cost partially explains why most early numerical investigations of thermal creep flows are focused on one-dimensional, semi-infinite or pseudo two-dimensional cases and used the linearized form of the kinetic equations. The thermal creep flows in an enclosure has also been served as a

\footnotetext{
*zlguo@hust.edu.cn
} 
benchmark problem for evaluating more efficient novel numerical methods such as the octant flux splitting information preservation (IP) DSMC method [24], the low-variance Monte Carlo simulation method [25], the regularized-13 (R13) equations method $[18,26]$, the (discrete) unified gas kinetic scheme (UGKS, DUGKS) $[19,27]$ and the fast spectral method for full Boltzmann equation [21, 28].

Based on the above literature review, it can be seen that previous studies on thermal creep flow in enclosures are all based on the two-dimensional cavity. To the best of the authors' knowledge, there is no numerical study on thermal creep flow in a three-dimensional enclosure due to the high computational cost using either the DSMC approach or the discrete velocity based deterministic methods. For rarefied flow in many realistic three-dimensional applications, as the gas-wall interaction becomes more significant, the extra confinement introduced by the walls in the third dimension has to be considered explicitly. Therefore, there is an urgent need to investigate the thermal creep flow in three-dimensional geometries.

In the present study, the thermal creep flow in a three-dimensional square channel with alternatively heated closed ends and linear temperature distribution along the lateral walls will be numerically investigated in a wide range of Knudsen numbers (Kn). Various temperature differences between the two enclosure ends and different aspect ratios are considered. The numerical method employed is a discrete velocity method (DVM) for the Shakhov kinetic equation [29]. A detailed grid independence study has been conducted in both spatial and velocity spaces. A recently proposed iterative scheme with the memory reduction technique [30] enables us to accurately describe the highly nonequilibrium velocity distribution function in very fine discrete velocity grids with moderate computational expense and small memory capacity requirement. Thus macroscopic variables such as the velocity field can be accurately obtained. The influences of the Knudsen number, temperature ratio and geometric aspect ratio on the flow field will then be analyzed.

The rest of the paper is organized as follows. In Sec. II, we describe in detail the problem and assumptions. In Sec. III, the governing equation and numerical method are introduced. Numerical results are given and analyzed in Sec. IV followed by the conclusions in Sec. V.

\section{SIMULATION SETUP}

The computational domain is a straight channel with a length of $L_{y}$ and a square cross-section of $L_{x} \times L_{z}\left(L_{x}=L_{z}\right)$ as illustrated in Fig. 1. Uniform temperatures, $T_{h}$ and $T_{c}$, are applied at two ends of the domain $\left(y=0\right.$ and $\left.y=L_{y}\right)$, respectively. The temperature on each lateral side wall varies linearly from $T_{h}$ at the left end $(y=0)$ to $T_{c}$ at the right end $\left(y=L_{y}\right)$. The gas in the enclose is assumed to be monatomic with a viscosity $\mu$ related to the temperature $T$ by $\mu=\mu_{0}\left(T / T_{0}\right)^{\omega}$, where $\mu_{0}$ is the reference viscosity at the reference temperature $T_{0}$. The exponential index $\omega$ is set to be 0.81 , corresponding to the variable hard sphere (VHS) model of argon gas at 273.15K [23]. The reference viscosity is defined using the VHS model [23, 31],

$$
\mu_{0}=\frac{15 \sqrt{\pi} p_{0} \lambda_{0}}{(5-2 \omega)(7-2 \omega) \sqrt{2 R T_{0}}}
$$

where $\lambda_{0}$ is the referenced mean free path and $R$ is specific gas constant, $p_{0}$ is the referenced pressure and is related to the reference density $\rho_{0}$ (the average gas density in the enclosure) by $p_{0}=\rho_{0} R T_{0}$. The Knudsen number of the system is defined by $\mathrm{Kn}=\lambda_{0} / L_{0}$ with $L_{0}$ being the reference length. The reference values are taken as $T_{0}=T_{h}$ and $L_{0}=L_{x}$. Furthermore, the walls are assumed to be purely diffusive.

We consider only the steady state which depends only on three non-dimensional parameters, $L_{y} / L_{x}, T_{c} / T_{h}$ and Kn. The Knudsen number range considered is $0.01 \leq \mathrm{Kn} \leq 10$. To investigate the influences of temperature ratio $\left(T_{c} / T_{h}\right)$ and aspect ratio $\left(L_{y} / L_{x}\right)$, three values of both $T_{c} / T_{h}$ and $L_{y} / L_{x}$ will be considered for each selected Knudsen number $(\mathrm{Kn}=0.1,1$ and 10). The flow field is numerically obtained by solving the steady state Shakhov model (S-model) equation using a discrete velocity method. The Shakhov model equation is appropriate for thermally driven flow as has been demonstrated by numerous applications in the literature, such as Refs. [19, 20, 32-34], to name a few. It should be noted that to match the realistic gas viscosity $[35,36]$ in a wide temperature range, the power-law index $\omega$ will vary significantly. However, the deviation of viscosity predicted by the VHS model with a fixed $\omega=0.81$ is still acceptable in the temperature range considered in this work. The recent study in Ref. [20] shows that the overall agreement between the flow fields predicted using the Shakhov model equation and the DSMC method of for the thermally driven flow can be quite good even the temperature field varies significantly in space. Details of the numerical method will be explained in the following section. 


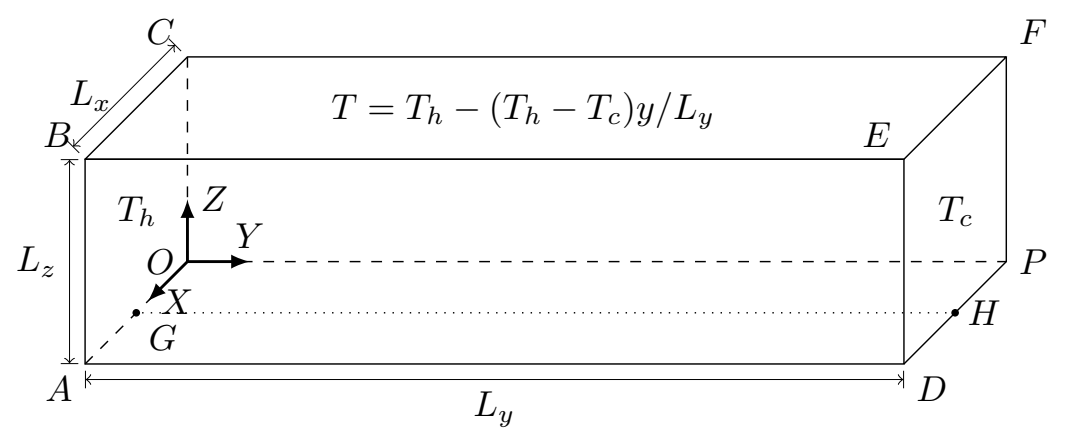

FIG. 1. Schematic of the square channel with differently heated closed ends and iso-thermal lateral walls. The left and right end sides (denoted by $O A B C$ and $P D E F$ ) are kept at higher and lower uniform temperatures $T_{h}$ and $T_{c}$, respectively. The temperature of the lateral sides varies linearly with respect to the $\mathrm{Y}$ coordinate $(y)$ from $T_{h}$ at the left to $T_{c}$ at the right. The dotted line $G H$ denotes the symmetry line of the lateral side $O A D P$, which will be referenced in numerical results section of the body text.

\section{GOVERNING EQUATION AND NUMERICAL METHOD}

\section{A. Governing equation}

In this study, we use the steady state Shakhov model equation [29] as the governing equation of the velocity distribution function $f(\boldsymbol{x}, \boldsymbol{\xi})$,

$$
\boldsymbol{\xi} \cdot \nabla f=-\frac{f-f^{S}}{\tau},
$$

where $\tau$ is the relaxation time and is related to the local viscosity $\mu$ and pressure $p$ by $\tau=\mu / p$ to recovery the correct viscosity [37]. $f^{S}$ is defined as

$$
f^{S}=f^{M}\left[1+(1-\operatorname{Pr}) \frac{\boldsymbol{c} \cdot \boldsymbol{q}}{5 p R T}\left(\frac{c^{2}}{R T}-5\right)\right], \quad \text { with } \quad f^{M}=\frac{\rho}{(2 \pi R T)^{3 / 2}} \exp \left(-\frac{c^{2}}{2 R T}\right),
$$

where Pr is the Prandtl number and $\boldsymbol{c}=\boldsymbol{\xi}-\boldsymbol{U}$ is the peculiar velocity with $\boldsymbol{U}$ being the hydrodynamic velocity. For a monatomic gas, Pr equals to $2 / 3$. The macroscopic variables such as the density $\rho$, velocity $\boldsymbol{U}$, temperature $T$, and heat flux $\boldsymbol{q}$ can be calculated from the moments of the distribution function,

$$
\rho=\int f \mathrm{~d} \boldsymbol{\xi}, \quad \rho \boldsymbol{U}=\int \boldsymbol{\xi} f \mathrm{~d} \boldsymbol{\xi}, \quad \rho E=\frac{1}{2} \int \xi^{2} \mathrm{~d} \boldsymbol{\xi}, \quad \boldsymbol{q}=\frac{1}{2} \int \boldsymbol{c} c^{2} f \mathrm{~d} \boldsymbol{\xi}
$$

where $\rho E=1 / 2 \rho U^{2}+C_{v} T$ is the total energy with $C_{v}$ being the heat capacity [(3/2)R for monatomic gases]. The pressure is related to the density and temperature by $p=\rho R T$.

Equation (2) can be written in a non-dimensional form as

$$
\hat{\boldsymbol{\xi}} \cdot \hat{\boldsymbol{\nabla}} \hat{f}=-\frac{1}{\hat{\tau}}\left[\hat{f}-\hat{f}^{S}\right]
$$

with

$$
\hat{f}^{S}=\hat{f}^{M}\left[1+\frac{4}{5}(1-\operatorname{Pr}) \frac{\hat{\boldsymbol{c}} \cdot \hat{\boldsymbol{q}}}{\hat{\rho} \hat{T}^{2}}\left(\frac{2 \hat{c}^{2}}{\hat{T}}-5\right)\right], \quad \hat{f}^{M}=\frac{\hat{\rho}}{(\pi \hat{T})^{3 / 2}} \exp \left(-\frac{\hat{c}^{2}}{\hat{T}}\right)
$$

where the non-dimensional variables are defined as

$$
\begin{array}{r}
\hat{\boldsymbol{\xi}}=\boldsymbol{\xi} / \sqrt{2 R T_{0}}, \hat{\boldsymbol{\nabla}}=L_{0} \boldsymbol{\nabla}, \hat{\rho}=\rho / \rho_{0}, \hat{c}=c / \sqrt{2 R T_{0}}, \hat{\boldsymbol{U}}=\boldsymbol{U} / \sqrt{2 R T_{0}}, \\
\hat{T}=T / T_{0}, \hat{\boldsymbol{q}}=\boldsymbol{q} /\left[\rho_{0}\left(2 R T_{0}\right)^{3 / 2}\right], \hat{f}=f\left(2 R T_{0}\right)^{3 / 2} / \rho_{0}, \hat{f^{S}}=f^{S}\left(2 R T_{0}\right)^{3 / 2} / \rho_{0} .
\end{array}
$$

$\hat{\tau}$ is calculated by $\hat{\tau}=\beta \hat{\rho}^{-1} \hat{T}^{(\omega-1)} \mathrm{Kn}$ where $\beta=15 \sqrt{\pi} /[(5-2 \omega)(7-2 \omega)]$. The hats on the non-dimensional variables will be omitted in the following analysis for convenience. 
In the fully diffusive wall model, the gas molecules hit the wall are assumed to be fully accommodated with the wall, and are re-emitted from the wall with an equilibrium distribution associated with the non-dimensional local wall temperature $T_{w}$,

$$
f=f^{M}\left(\rho_{w}, 0, T_{w}\right) \text { for } \boldsymbol{\xi} \cdot \boldsymbol{n}_{w}>0
$$

where $\boldsymbol{n}_{w}$ is the unit normal vector of the wall pointing to the flow domain, and $f^{M}\left(\rho_{w}, 0, T_{w}\right)$ stands for the equilibrium distribution function of gas molecular with velocity $\boldsymbol{\xi}$ and the arguments of density $\rho_{w}$, temperature $T_{w}$, and zero wall velocity. The density $\rho_{w}$ is determined by the impermeable wall condition [37], i.e.,

$$
\rho_{w}=-\sqrt{\frac{2 \pi}{R T_{w}}} \int_{\boldsymbol{\xi} \cdot \boldsymbol{n}_{w}<0} f \boldsymbol{\xi} \cdot \boldsymbol{n}_{w} \mathrm{~d} \boldsymbol{\xi}
$$

The velocity space is first discretized in the particle velocity space with a chosen three-dimensional discrete velocity grid $\left\{\boldsymbol{\xi}_{\alpha} \mid \alpha=1,2, \ldots, M\right\}$. The discrete form of the Shakhov equation [Eq. (2)] is then expressed as

$$
\boldsymbol{\xi}_{\alpha} \cdot \nabla f_{\alpha}=-\frac{1}{\tau}\left[f_{\alpha}-f_{\alpha}^{S}\right]
$$

where $f_{\alpha}$ and $f_{\alpha}^{S}$ are the distribution function and equilibrium distribution function with discrete velocity $\boldsymbol{\xi}_{\alpha}$. The macro variables are evaluated by taking numerical moments of $f_{\alpha}$ as follows,

$$
\rho=\sum_{\alpha} w_{\alpha} f_{\alpha}, \rho \boldsymbol{U}=\sum_{\alpha} w_{\alpha} \boldsymbol{\xi}_{\alpha} f_{\alpha}, \rho E=\frac{1}{2} \sum_{\alpha} w_{\alpha}\left(\xi_{\alpha, x}^{2}+\xi_{\alpha, y}^{2}+\xi_{\alpha, z}^{2}\right) f_{\alpha}, \boldsymbol{q}=\frac{1}{2} \sum_{\alpha} w_{\alpha} \boldsymbol{c}_{\alpha}\left(c_{\alpha, x}^{2}+c_{\alpha, y}^{2}+c_{\alpha, z}^{2}\right) f_{\alpha}
$$

where $\omega_{\alpha}$ are the weight coefficient for the numerical quadrature. In practice, the discrete velocity set and the corresponding numerical quadrature rules have to be carefully chosen to accurately capture the non-equilibrium distribution function while keeping the overall computational cost manageable. A grid independence study in the velocity space has been conducted in order to choose the optimized discrete velocity set. The detail results and comparisons are presented in the Appendix. For $\mathrm{Kn}<1$, we use the half-range Gauss-Hermit quadrature; For higher Knudsen number $\mathrm{Kn} \geq 1$, we use the following nonuniform discrete velocity set in each direction [21, 38],

$$
\xi_{i}=\frac{\xi_{\max }}{(N-1)^{3}}(1-N+2 i)^{3}, \quad i=0,2, \ldots, N-1
$$

and use the trapezoidal rule for the numerical quadrature. $\xi_{\max }$ is the maximal discrete velocity component and is set to be 5 in the current study. $N$ is the number of discrete velocities in each axis direction and is fixed at $N=64$ (see the Appendix).

\section{B. Numerical schemes}

We apply a recently developed iterative scheme [30] to solve the above discrete Shakhov equation [Eq. (10)]. The novelty of the iterative scheme is that it permits us to store the distribution function of only one discrete velocity instead of the whole set of discrete velocities, which can reduce the total memory requirement dramatically [30]. Thus, sufficiently large velocity grid can be used with minimum computing resource requirement. Its efficiency for rarefied internal gas flows has already been demonstrated previously [30]. We will only brief the algorithm here.

Equation (10) is solved using the following iterative method,

$$
\boldsymbol{\xi}_{\alpha} \cdot \nabla f_{\alpha}^{n+1}=-\frac{1}{\tau^{n}}\left[f_{\alpha}^{n+1}-f_{\alpha}^{S, n}\right]
$$

At the beginning of the iteration, i.e., when $n=0, f_{\alpha}^{S, 0}$ is calculated from the guessed initial macro variables fields. From Eq. (13) we can see there is a one-to-one correspondence between $f^{S, n}$ and $f^{n+1}$. That is to say, for a given macro field and hence the equilibrium distribution function $f^{S, n}$, we can get the distribution function $f^{n+1}$ that satisfies Eq. (13). By solving for each discrete velocity, the guessed macro field can be corrected from the updated $f^{n+1}$. $f^{S, n+1}$ can also be updated accordingly before the next iteration being performed.

If the advection term is discretized directly with the second order central scheme, it will result a linear system to be solved. To avoid solving a linear system, but still attain a second order accuracy, Eq. (13) is rewritten as the following delta form,

$$
\boldsymbol{\xi}_{\alpha} \nabla \Delta f_{\alpha}^{n+1}+\frac{\Delta f_{\alpha}^{n+1}}{\tau^{n}}=\boldsymbol{\xi}_{\alpha} \nabla f_{\alpha}^{n}-\frac{1}{\tau^{n}}\left[f_{\alpha}^{n}-f_{\alpha}^{S, n}\right]
$$


where $\Delta f_{\alpha}^{n+1} \equiv f_{\alpha}^{n+1}-f_{\alpha}^{n}$. The gradients on the left and right hand sides are discretized using the first order upwind scheme and the second order central scheme respectively. Thus the second order accuracy at the ending of the iterating process will be achieved since $\Delta f_{\alpha}^{n+1}$, and accordingly, the left hand side of Eq. (14) will eventually approach to zero. Due to the first order upwind discretization of the implicit part in Eq. (14), it can be solved using a space sweeping algorithm, which is much simpler than solving a linear system resulting from applying a second-order discretization directly on Eq. (13), especially for higher dimension cases. The iteration stops when the relative changes in the $L_{2}$ norms of the density, temperature, velocity and heat flux fields between two consecutive steps are less than $10^{-9}$.

Since we store the distribution function of only one discrete velocity, when solving Eq. (14) for a specific discrete velocity from $n$ to $n+1, f^{n}$ is not known a priori. We solve this problem by using an inner iteration inside the step from $n$ to $n+1$, which is now called the outer iteration step [30]. The inner iteration step index is denoted by $m$. The guessed initial value of the distribution function in the inner iteration is $f^{n, 0}=f^{S, n}$. The inner iteration updating rule is

$$
\boldsymbol{\xi}_{\alpha} \boldsymbol{\nabla} \Delta f_{\alpha}^{n, m+1}+\frac{\Delta f_{\alpha}^{n, m+1}}{\tau^{n}}=\boldsymbol{\xi}_{\alpha} \nabla f_{\alpha}^{n, m}-\frac{1}{\tau^{n}}\left[f_{\alpha}^{n, m}-f_{\alpha}^{S, n}\right]
$$

where $\Delta f_{\alpha}^{n, m+1} \equiv f_{\alpha}^{n, m+1}-f_{\alpha}^{n, m}$. At the end of inner iteration, the distribution function is assigned to the new step value of the outer iteration, i.e., $f^{n+1}:=f^{n, M}$, where $M$ denotes the end step for the inner iteration and is determined by the convergence criterion. We found the convergence criterion for the inner iteration is not as important as the outer iteration. We set $M=10$ for all of the cases if not stated otherwise. It should be noted that, introducing of the inner iteration is merely a requirement to employ the memory reduction technique and achieve a second-order physical space accuracy at the same time. Compared with the conventional iterative DVM schemes, such a treatment will lead to much longer computing time (depends on $M$ ). But this memory reduction technique enables us to simulate a large problem on a smaller number of nodes. In addition, we can initially use first-order upwind scheme on both side of Eq. (14), then the inner iteration can converge in a single step [30]. This efficient first-order scheme can be used to obtain a first-order solution of the macro fields quickly as an initial solution for the later run using second-order scheme.

Equation (15) is discretized in the physical space using a cell-centered finite volume method. Linearly stretched meshes are used with the smallest cell distributed near the walls. Due to the two-fold symmetry of the problem in the $\mathrm{X}-\mathrm{Z}$ plane, we only simulate a quarter of the full domain. For the detailed configuration of the meshes, the reader is referred to the Appendix, where the grid independence verification in the spatial space has also been conducted. As the gradient at the left hand side of Eq. (15) is discretized using the first order upwind scheme, the updating of the distribution function can be executed sequentially among the cells along the direction of the discrete velocity. The spatial space sweeping always starts from a corner, and the wall distribution function is linearly extrapolated from the old step distribution function at interior of the flow domain. The diffusively reflected distribution function can be then calculated as

$$
f_{\alpha}^{n, m+1}=f_{\alpha}^{M}\left(\rho_{w}^{n, m}, 0, T_{w}\right) \text { for } \quad \boldsymbol{\xi}_{\alpha} \cdot \boldsymbol{n}_{w}>0,
$$

where $\rho_{w}^{n, m}$ is calculated from [37]

$$
\rho_{w}^{n, m}=-\sqrt{\frac{2 \pi}{R T_{w}}} \sum_{\xi_{\alpha} \cdot \boldsymbol{n}_{w}<0} f_{\alpha}^{n, m} \boldsymbol{\xi}_{\alpha} \boldsymbol{n}_{w} .
$$

Due to the mismatch of $m$ and $m+1$ in Eq. (16), the total mass in the flow domain is not conserved from step $n$ to step $n+1$. We fix this problem by simply scaling the density of the whole field by a constant after each outer iteration step such the total mass is unchanged. The scale constant will converge to 1 as the outer iteration converges.

The above numerical method is implemented using $\mathrm{C}++$ language and is parallelized in the velocity space with the Message Passing Interface (MPI) library. The solver runs on Tianhe-2 supercomputing system with each compute node equipped with two 12-core Intel Xeon E5-2695v2 (Ivy Bridge EP) CPUs and 64 GB of main memory. As an indication of the overall computing cost, we listed the computing time and configurations of typical cases with $\mathrm{Kn}=0.01,0.1,1$ and10 in Table I. Note that without the memory reduction technique [30], the 4 nodes' (96 MPI processes in total) memory is not enough to hold the distribution function arrays for the cases with velocity grid size of $64^{3}$. It can bee seen that the current discretization scheme converges significantly slower for the low Kn cases. This is because $f^{S}$ in Eq. (13) is treated explicitly. We note there have been more efficient asymptotical preserving schemes and implicit schemes developed recently that overcome this issue, e.g. the (implicit) (D)UGKS [39-42]. 
TABLE I. Computing time for typical cases.

\begin{tabular}{llllll}
\hline \hline Kn & $\begin{array}{l}\text { physical } \\
\text { grid size }\end{array}$ & $\begin{array}{l}\text { velocity } \\
\text { grid size }\end{array}$ & $\begin{array}{l}\text { num. of outer } \\
\text { iterations }\end{array}$ & $\begin{array}{l}\text { num. of MPI } \\
\text { processes }\end{array}$ & $\begin{array}{l}\text { computing time } \\
\text { (wall time) }\end{array}$ \\
\hline 0.01 & $40 \times 40 \times 80$ & $16^{3}$ & 11322 & 96 & $7.4 \mathrm{~h}$ \\
0.1 & $40 \times 40 \times 80$ & $28^{3}$ & 673 & 96 & $1.6 \mathrm{~h}$ \\
1 & $40 \times 40 \times 80$ & $64^{3}$ & 296 & 96 & $7.0 \mathrm{~h}$ \\
10 & $40 \times 40 \times 80$ & $64^{3}$ & 411 & 96 & $11.4 \mathrm{~h}$ \\
\hline \hline
\end{tabular}

\section{RESULTS AND DISCUSSION}

The flow fields simulated under different configurations are presented and analyzed in this section. The Knudsen number range considered is $[0.01,10]$, covering from the early slip flow regime to the early free-molecular flow regime. We also considered different temperature ratios $\left(T_{c} / T_{h}=0.5,0.8\right.$ and 0.9$)$ between the hot and cold ends and also different channel aspect ratios $\left(L_{y} / L_{x}=5,2\right.$ and 1) at three typical Knudsen numbers $(\mathrm{Kn}=0.1,1$ and 10). When analyzing the effect of each parameter, we keep the other parameters fixed at the base configuration which is $T_{c} / T_{h}=0.5$ and $L_{y} / L_{x}=5$.
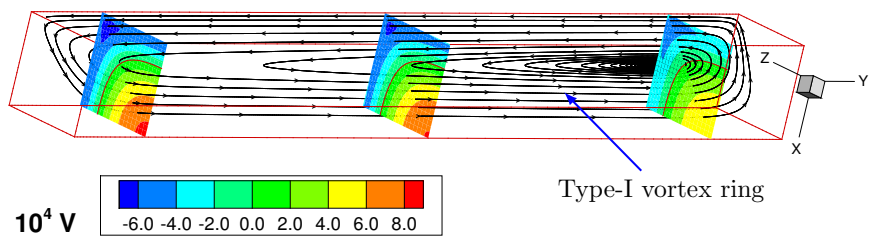

(a) $\mathrm{Kn}=0.01$
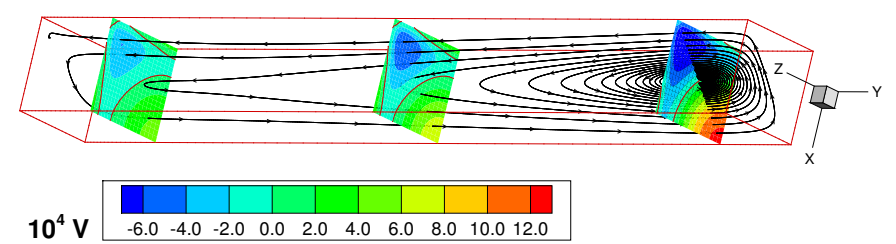

(c) $\mathrm{Kn}=0.2$

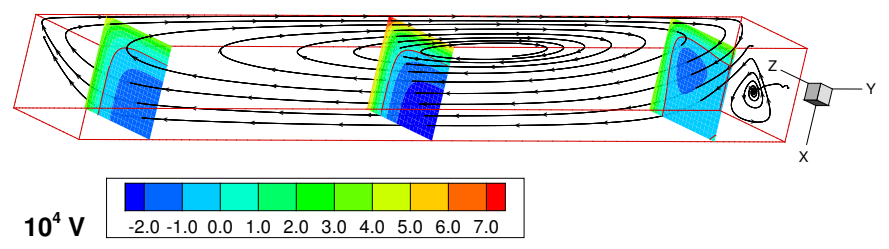

(e) $\mathrm{Kn}=1$
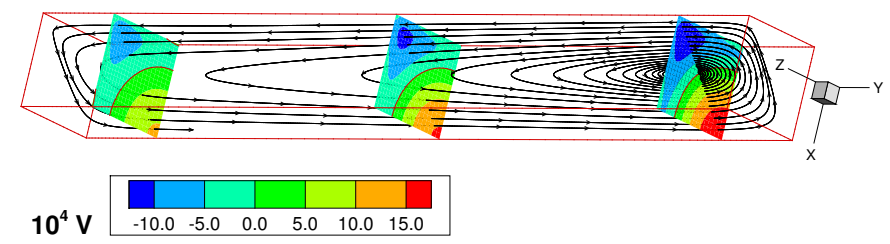

(b) $\mathrm{Kn}=0.1$

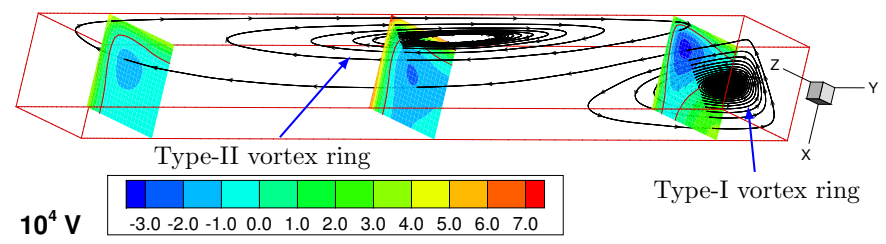

(d) $\mathrm{Kn}=0.5$

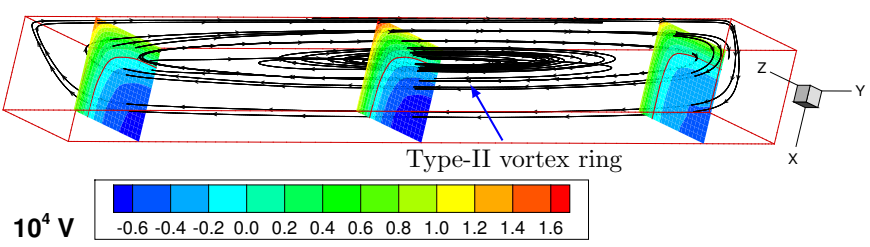

(f) $\mathrm{Kn}=10$

FIG. 2. Streamlines and sliced contour plots of V-velocity at various Knudsen numbers. Only one quarter of the domain $(x \in[0,0.5], z \in[0.5,1])$ is shown due to the two-fold symmetry in $\mathrm{X}$ and $\mathrm{Z}$ directions. In each sub-figure, the sliced contour plots are at positions of $\mathrm{y}=0.5,2.5$ and 4.5. In each contour plots, the red solid line(s) indicate(s) the contour level of $V=0$. The streamlines are only shown in the diagonal plane. All cases are based on the base temperature and geometry configuration, i.e., $T_{c} / T_{h}=0.5$ and $L_{y} / L_{x}=5$.

\section{A. General flow patterns}

The effect of gas rarefaction to the overall flow field features is shown in Fig. 2, where the velocity streamlines and slices along the $\mathrm{Y}$ direction with $\mathrm{V}$-velocity contours are presented for the cases of different Knudsen numbers. The figures show that the flow field changes significantly as Kn increases from 0.01 to 10 . 
By comparing the two extreme cases, i.e., $\mathrm{Kn}=0.01$ and $\mathrm{Kn}=10$, we can find that, in both cases, there is a vortex ring developed in the entire space of the channel, and the slip flow develops on the lateral walls. However, the flow directions of the vortex ring or the slip flow are different in the two cases. For the case of $\mathrm{Kn}=0.01$, the slip flow near the wall is from the cold to hot end (right to left in the figures), which can be explained by the thermal creep flow theory. While for $\mathrm{Kn}=10$, the slip flow is from the hot to the cold end. It is noted that such opposite results have been observed in previous two-dimensional configurations [18-20], and is explained by the balance between the shear stress and tangential heat flux induced driven mechanisms $[18,20,26]$. We denote the vortex ring in the case of $\mathrm{Kn}=0.01$ as type-I and in the case of $\mathrm{Kn}=10$ as type-II for convenience in the following discussions [see the labels in Fig. 2(a), (d) and (f)].

From the flow fields at the intermediate Kn presented in Fig. 2, we can find how the type-I and type-II vortex rings evolve as the gas is getting more rarefied. At $\mathrm{Kn}=0.1$, the cold-to-hot flow region expands compared with the case of $\mathrm{Kn}=0.01$, and the overall magnitude of velocity is bigger. But the hot-to-cold slip flow can be observed on the lateral walls near the hot end [see the sliced plots in Fig. 2(b)]. These small regions of hot-to-cold flow suggest that four eddies have begun to develop there. As $\mathrm{Kn}$ increases to 0.2 , the overall flow is getting weaker. The hot-to-cold slip flow region spreads to the majority area of later walls and even appears in the vicinity of the lateral edges in the cold part. At $\mathrm{Kn}=0.5$, the flow in the inner region gets further weaker. The hot-to-cold slip flow has developed on almost the entire lateral walls and is getting stronger. The type-II vortex ring has developed from the four discrete eddies and coexists with the type-I vortex ring. The type-I vortex ring is now constrained in a much smaller region. As Kn increases to 1, the type-II vortex ring dominates the flow, while the type-I vortex ring resides only near the cold end. The overall flow slows down but the flow near the lateral edges becomes stronger. In the limiting case, i.e., $\mathrm{Kn}=10$, the type-I vortex ring disappears and the type-II vortex ring is fully developed.

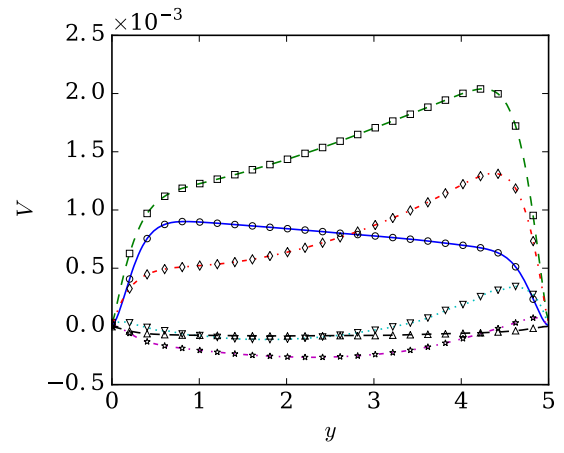

(a)

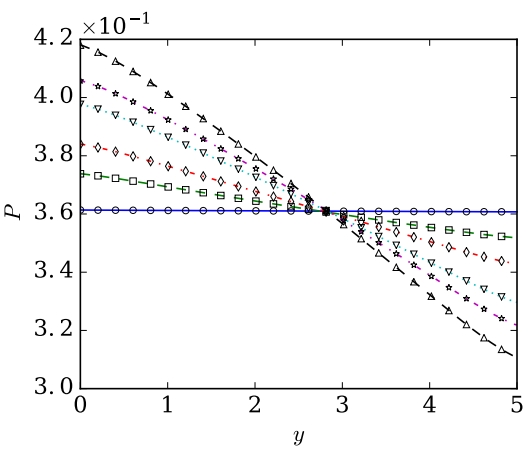

(b)

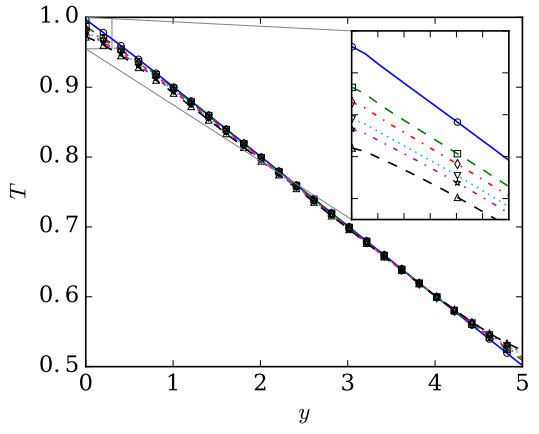

(c)

FIG. 3. V-velocity (a), pressure (b) and temperature (c) profiles on the geometric center line of the channel along the Y

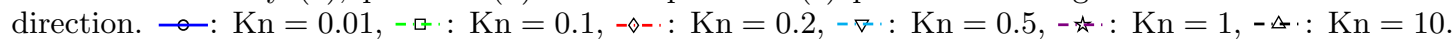

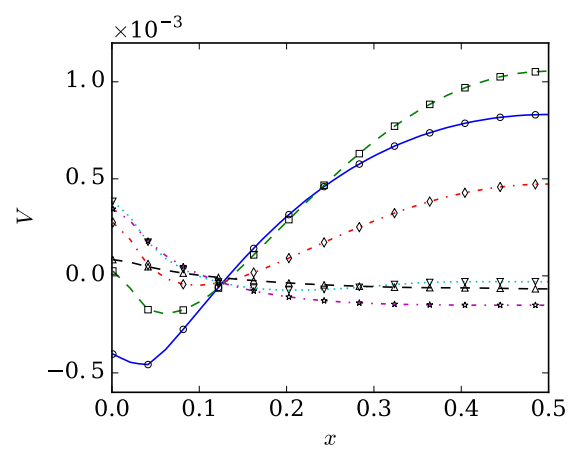

(a)

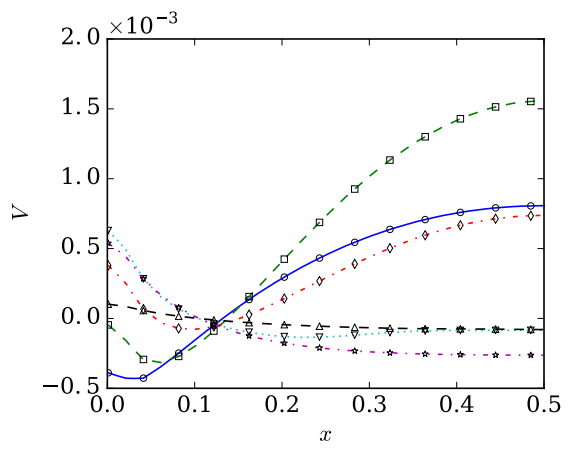

(b)

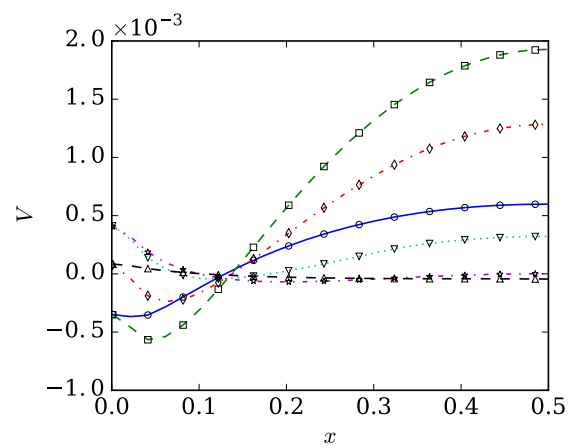

(c)

FIG. 4. V-velocity profiles on the X-direction center lines of the slices shown in Fig. 2, i.e., slices at positions of $y=0.5$ (a),

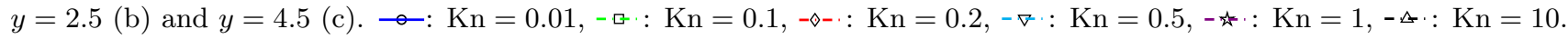


TABLE II. Average heat flux $\bar{q}_{y}$ on the hot (left) and cold (right) sides at various Knudsen numbers. All cases are based on the base temperature and geometry configuration, i.e., $T_{c} / T_{h}=0.5$ and $L_{y} / L_{x}=5$.

\begin{tabular}{lll}
\hline \hline Kn & Hot side & Cold side \\
\hline 0.1 & $5.573 \times 10^{-3}$ & $4.729 \times 10^{-3}$ \\
0.2 & $7.779 \times 10^{-3}$ & $7.150 \times 10^{-3}$ \\
0.5 & $1.073 \times 10^{-2}$ & $1.060 \times 10^{-2}$ \\
1 & $1.264 \times 10^{-2}$ & $1.280 \times 10^{-2}$ \\
10 & $1.594 \times 10^{-2}$ & $1.607 \times 10^{-2}$ \\
\hline \hline
\end{tabular}
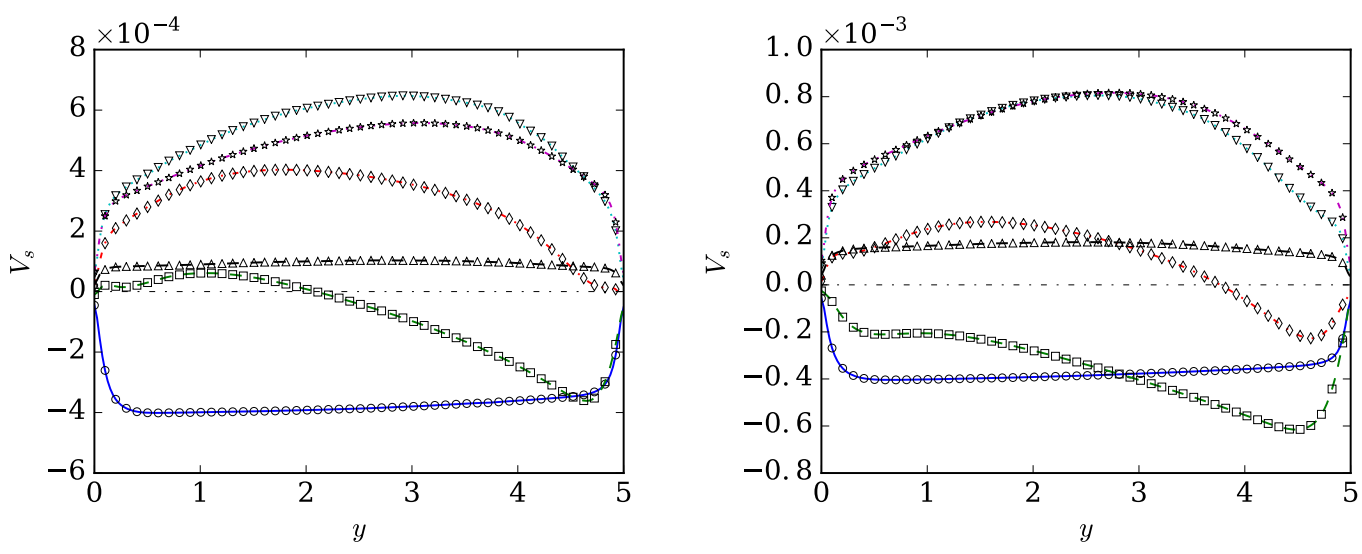

FIG. 5. V-slip-velocity profiles on the Y-direction center line of the lateral walls (a) and the lateral edges (b). See the lines $G H$ and $O P$ in Fig. 1 for the profiles' location. The dashed black lines without symbols indicate the zero-velocity level. $\rightarrow-$ :

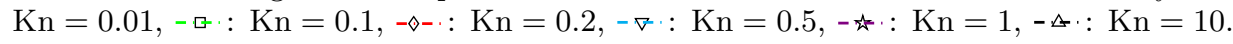

The V-velocity profiles in Figs. 3 and 4 provide more quantitative descriptions of the flow. Specifically, Figs. 3(a)(c) present the $\mathrm{V}$-velocity, pressure and temperature profiles on the geometric center line of the channel along the $\mathrm{Y}$ direction. Figure 3(a) shows that the maximum velocity is achieved at $\mathrm{Kn}=0.1$. It is also in accordance with the streamline plots in Fig. 2 that the hot-to-cold flow in the center region of the channel only occurs in cases of $\mathrm{Kn}<0.5$. For cases of $\mathrm{Kn} \geq 0.5$, the flow direction is reversed, even though Fig. 3(b) indicates the pressure ratio between the cold and hot ends accumulates with the increase of Kn. Figure 3(c) shows that as Kn increases, both the temperature jump and the slight non-linearity of the temperature distribution near the channel ends is more profound.

Figures 4(a)-(c) present the V-velocity profiles on the center lines in the $\mathrm{X}$ direction of the slices shown in Fig. 2. We can observe that the spatial variation of the flow field on the cross-sections is more significant for the low Knudsen number cases. Nevertheless, the variation near the wall is rapid for all of Kn considered. The results also suggest that the maximum velocity appears at $\mathrm{Kn}=0.1$.

To examine the near wall slip flow in detail, we extract the V-component of the slip velocity profiles on the Ydirection center line of the lateral sides and lateral edges, which are shown in Figs. 5(a) and 5(b), respectively (See the line $G H$ and $O P$ in Fig. 1 for the profiles' location). It can be seen that the slip velocity on the later edge is larger than that on the lateral-face center lines for each Kn generally, except for the case of $\mathrm{Kn}=0.01$, where the two profiles are very close to each other. Figure 5 also shows that the hot-to-cold flow develops quickly as Kn increases from 0.01 to 0.2 and appears at the hot side of the lateral walls as early as $\mathrm{Kn}=0.1$.

To investigate the heat transfer characteristics of the problem at different flow regimes, the average heat fluxes $\bar{q}_{y}$ on the hot and cold ends of the channel are calculated and listed in Table II. The net heat flux from the two ends are not zero as heat transfer occurs at the lateral walls. It can be seen that the heat transfer at both ends are enhanced rapidly with the increase of $\mathrm{Kn}$ in the slip and early transition regimes, while they increase only slightly after $\mathrm{Kn}>1$. It is also interesting to compare the average heat flux differences at the hot and cold ends. Table II shows the relative difference of the average heat fluxes from the two ends drops quickly in the slip and early transition regimes. For cases of $\mathrm{Kn} \geq 0.5$, the relative difference is only several percentages, indicating the total heat transfer from the lateral sides is relatively small. 


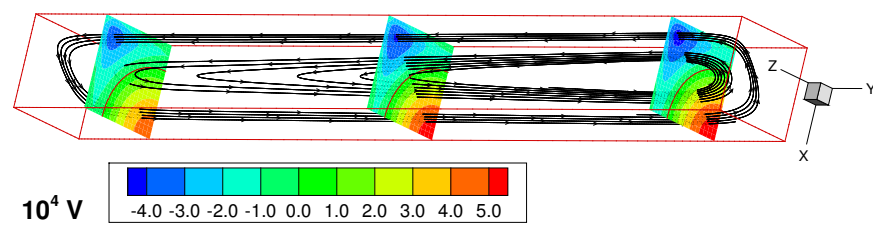

(a) $\mathrm{Kn}=0.1, T_{c} / T_{h}=0.8$

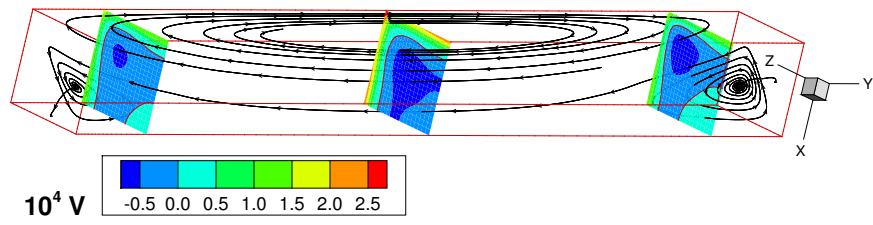

(c) $\mathrm{Kn}=0.5, T_{c} / T_{h}=0.8$

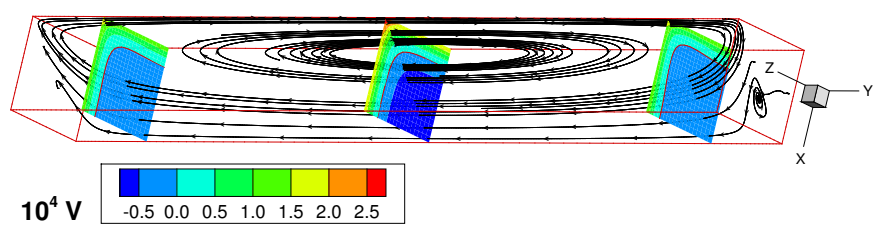

(e) $\mathrm{Kn}=1, T_{c} / T_{h}=0.8$

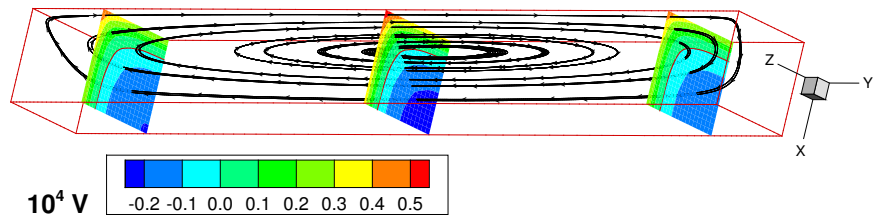

(g) $\mathrm{Kn}=10, T_{c} / T_{h}=0.8$

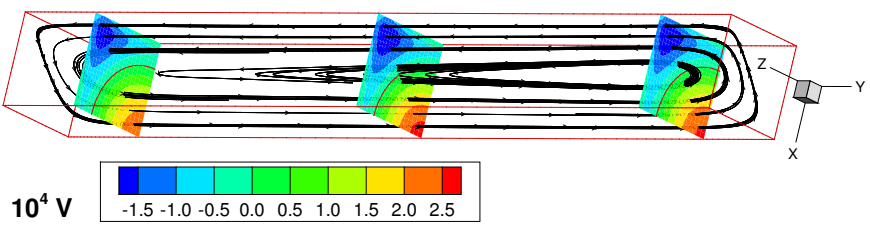

(b) $\mathrm{Kn}=0.1, T_{c} / T_{h}=0.9$

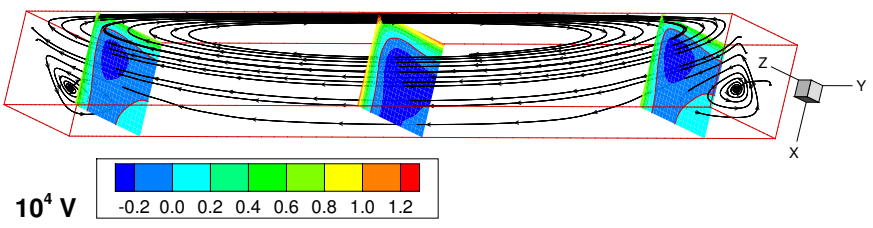

(d) $\mathrm{Kn}=0.5, T_{c} / T_{h}=0.9$

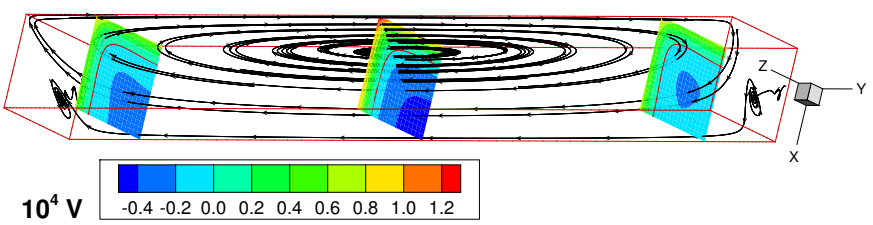

(f) $\mathrm{Kn}=1, T_{c} / T_{h}=0.9$

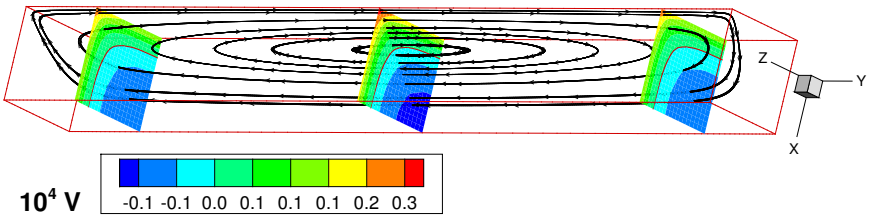

(h) $\mathrm{Kn}=10, T_{c} / T_{h}=0.9$

FIG. 6. Streamlines and sliced contour plots of V-velocity at different Knudsen numbers and temperature ratios. Only one quarter of the domain $(x \in[0,0.5], z \in[0.5,1])$ is shown due to the two-fold symmetry in $\mathrm{X}$ and $\mathrm{Z}$ directions. In each sub-figure, the contour plots are on slices at positions of $\mathrm{y}=0.5,2.5$ and 4.5. In each contour plots, the red solid line(s) indicate(s) the contour level of 0 . The streamlines are only shown in the two diagonal planes. The geometric aspect ratio $L_{y} / L_{x}$ is set as the base configuration, i.e., $L_{y} / L_{x}=5$.

\section{B. Effect of the temperature ratio}

The temperature ratio between the hot and cold ends is an important factor to the flow field since it is the nonuniformity of the temperature field that induces the flow. We consider the effect of the temperature ratio by simulating the problem with another two different temperature ratios, $T_{c} / T_{h}=0.8$ and 0.9 , other than the 0.5 used above. For each temperature ratio, the flow fields at $\mathrm{Kn}=0.1,1,0.5$ and 10 are obtained. It is worthwhile to mention that, for thermally induced rarefied flows, a larger temperature ratio means stronger non-equilibrium effect, and the DVM requires a fine discrete velocity grid to accurately predict the extremely slow velocity field. To make the full three-dimensional computational cost manageable, only moderate temperature ratios are considered here.

The overall flow fields at $T_{c} / T_{h}=0.8$ and 0.9 are shown in Fig. 6 . The flow fields at $T_{c} / T_{h}=0.5$ are not presented in parallel since they are already shown in Fig. 2. As expected, from Figs. 6 and 2, we can observe that the overall velocity magnitude decreases with the reducing of temperature difference. The results also show that the spatial variation of the flow field along the $\mathrm{Y}$ direction becomes smaller as the temperature ratio approaches to 1 . From the case of $\mathrm{Kn}=0.1$, it can be clearly seen that the type-I vortex ring is elongated almost to the entire channel length as the temperature increase to 0.9 . However, the results at $\mathrm{Kn}=1$ also show the type-I vortex ring is further constrained to an even smaller region near the cold end as $T_{c} / T_{h}$ increases from 0.5 to 0.8 .

Figures 7 and 8 present the V-velocity profiles along the Y-direction center line of the channel and the X-direction 


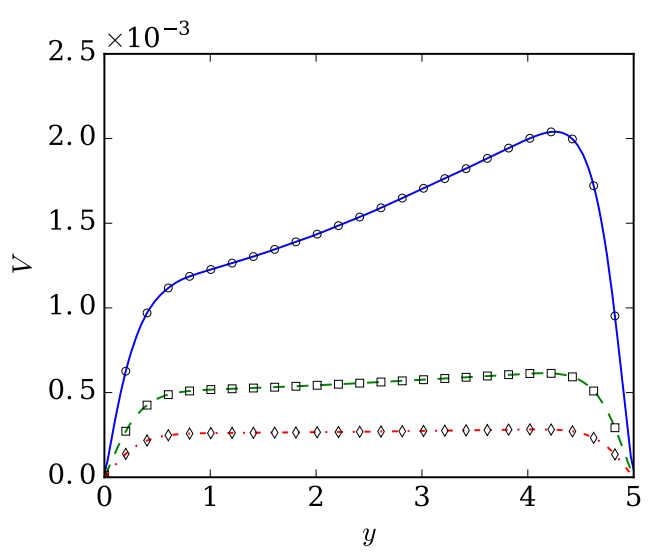

(a)

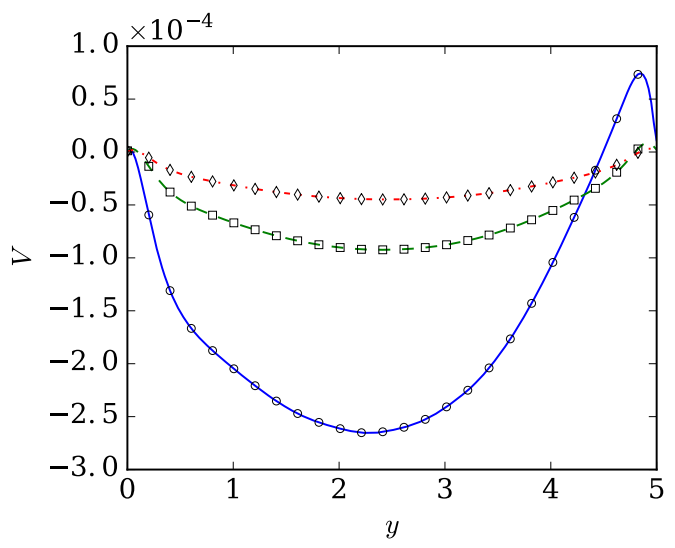

(c)

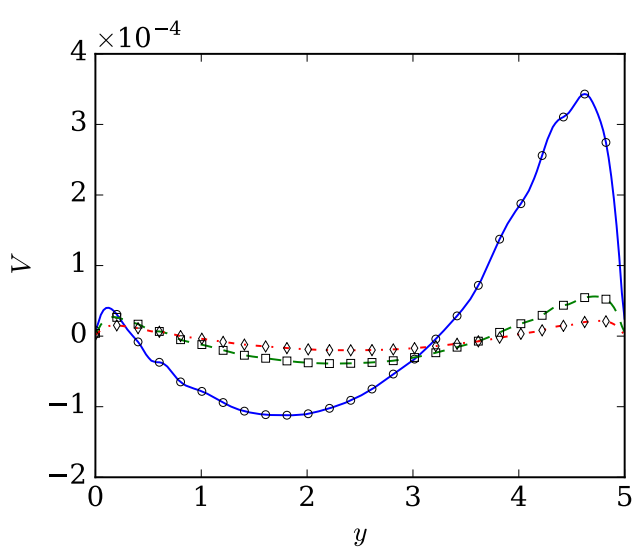

(b)

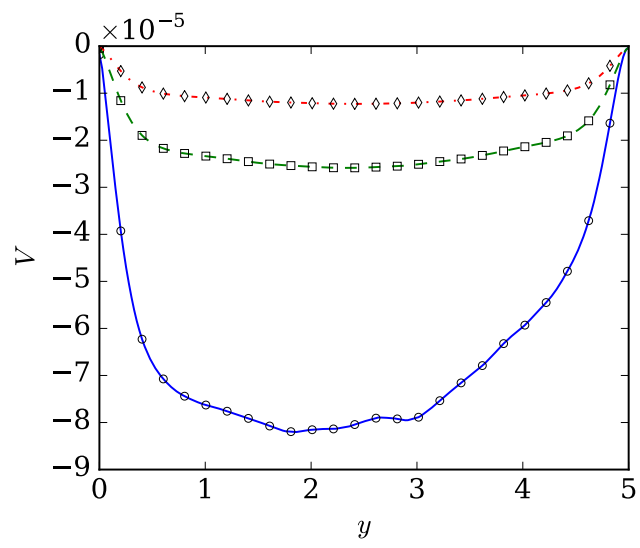

(d)

FIG. 7. V-velocity profiles on the Y-direction center lines of the geometry. (a) $\mathrm{Kn}=0.1$, (b)Kn $=0.5$, (c) Kn $=1$, (d) $\mathrm{Kn}=10$. In each sub-figure, different lines represent different temperature ratios $\left(\multimap-: T_{c} / T_{h}=0.5,-\mathrm{c}: T_{c} / T_{h}=0.8,-\diamond-\cdot:\right.$ $\left.T_{c} / T_{h}=0.9\right)$. The geometric aspect ratio $L_{y} / L_{x}$ is set as the base configuration, i.e., $L_{y} / L_{x}=5$.

center lines of the slices shown in Fig. 6, respectively for each Kn. The plots show that both the bulk and slip velocity become smaller as the temperature difference decreases, which confirm the observation in Fig. 6 that the spatial variation of the velocity field reduces as the temperature ratio approaches to 1 . For the case of $\mathrm{Kn}=10$ and $T_{c} / T_{h}=0.5$, some un-physical wrinkles appear in the center region of the channel as shown in Figs. 7(c) and 8(c), which can be attributed to the typical ray effect caused by the numerical error as the discrete velocity grid cannot fully resolve the highly non-equilibrium distribution function.

The average heat fluxes on the hot and cold ends at different temperature ratios are listed in Table III. For each $\mathrm{Kn}$, the average heat fluxes from the ends clearly drop as the temperature difference decreases, and drop quickly as the temperature ratio increases from 0.8 to 0.9 . It is also observed that at $\mathrm{Kn}=0.1$, the relative differences between the average heat fluxes at the hot and cold ends reduces clearly with the decrease of the temperature difference. The observations indicate that the heat and momentum transfer are strongly coupled for such flow.

\section{Effect of the geometric aspect ratio}

The effect of the geometric aspect ratio of the enclosure is also investigated. As the temperature gradient is imposed along the $\mathrm{Y}$ direction, the ratio $L_{y} / L_{x}$ has a much larger effect on the flow field than the other aspect ratio, i.e., $L_{z} / L_{x}$. Thus we only study the cases corresponding to different $L_{y} / L_{x}$ while keeping $L_{x}=L_{z}$. The values of $L_{y} / L_{x}$ considered are 5,2 and 1. For each aspect ratio, the flow field at $\mathrm{Kn}=0.1,1$ and 10 are obtained. The overall flow fields for the case of $L_{y} / L_{x}=5$ have already been presented in Fig. 2, while those for the cases of $L_{y} / L_{x}=2$ and 1 


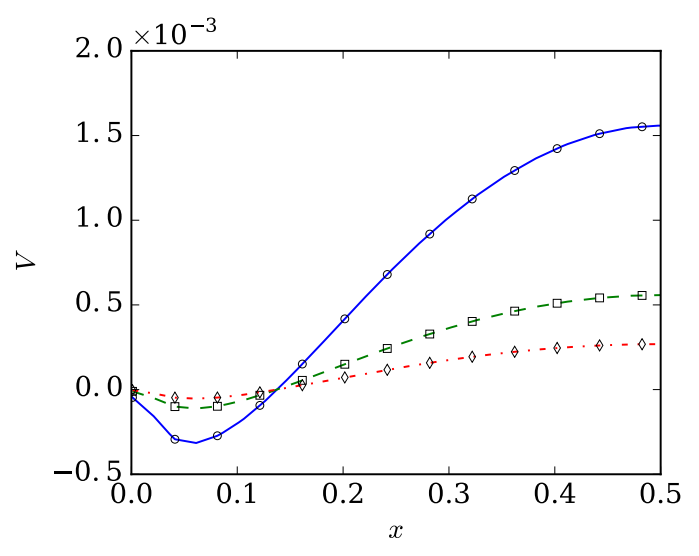

(a)

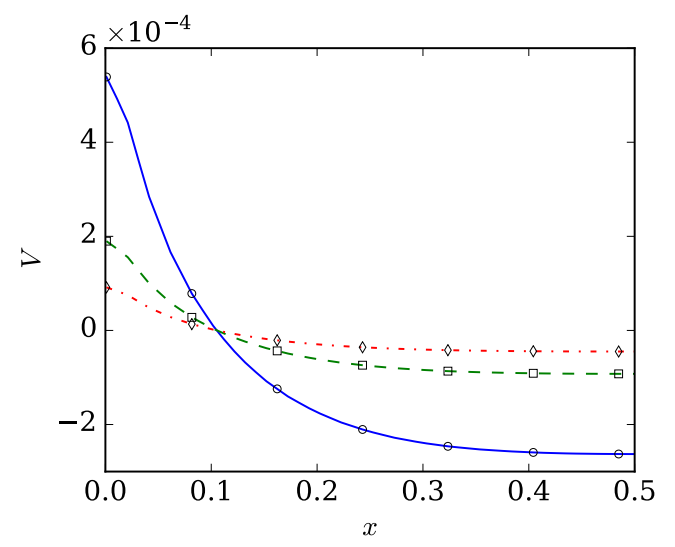

(c)

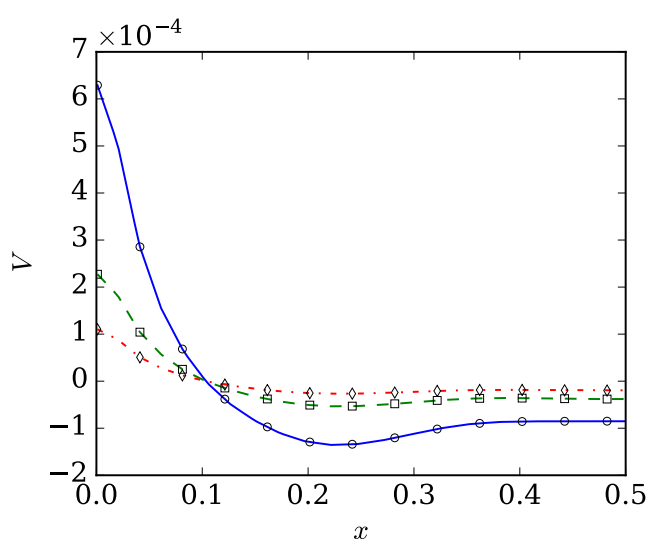

(b)

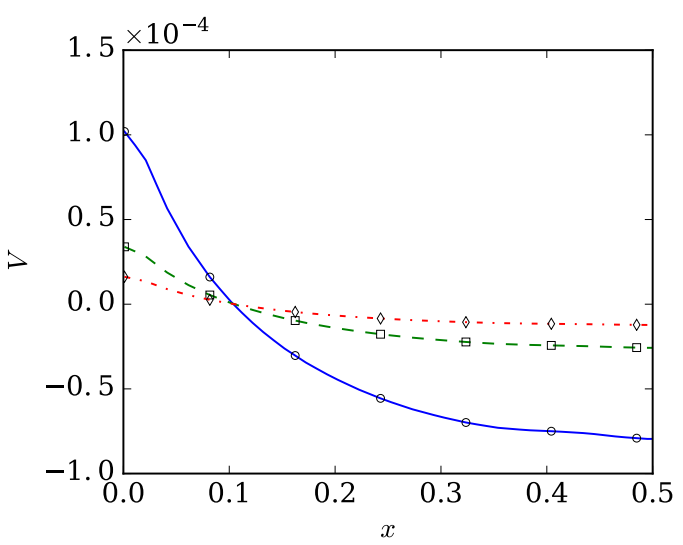

(d)

FIG. 8. V-velocity profiles on the X-direction center lines of the slices shown in Fig. 6. (a) $\mathrm{Kn}=0.1$, (b) Kn =0.5, (c) Kn = 1, (d) $\mathrm{Kn}=10$. In each sub-figure, different lines represent different temperature ratios $\left(\rightarrow-: T_{c} / T_{h}=0.5,-\mathrm{c}: T_{c} / T_{h}=0.8\right.$, $\left.-\diamond-\cdot T_{c} / T_{h}=0.9\right)$. The geometric aspect ratio $L_{y} / L_{x}$ is set as the base configuration, i.e., $L_{y} / L_{x}=5$.

TABLE III. Average heat flux $\bar{q}_{y}$ of the hot (left) and cold (right) ends at various Knudsen numbers and temperature ratios. The geometric aspect ratio $L_{y} / L_{x}$ is set as the base configuration, i.e., $L_{y} / L_{x}=5$.

\begin{tabular}{llll}
\hline \hline Kn $T_{c} / T_{h}$ & Hot side & Cold side \\
\hline 0.1 & 0.5 & $5.573 \times 10^{-3}$ & $4.729 \times 10^{-3}$ \\
& 0.8 & $2.465 \times 10^{-3}$ & $2.345 \times 10^{-3}$ \\
& 0.9 & $1.266 \times 10^{-3}$ & $1.236 \times 10^{-3}$ \\
0.5 & 0.5 & $1.073 \times 10^{-2}$ & $1.060 \times 10^{-2}$ \\
& 0.8 & $4.851 \times 10^{-3}$ & $4.838 \times 10^{-3}$ \\
& 0.9 & $2.507 \times 10^{-3}$ & $2.504 \times 10^{-3}$ \\
1 & 0.5 & $1.264 \times 10^{-2}$ & $1.280 \times 10^{-2}$ \\
& 0.8 & $5.718 \times 10^{-3}$ & $5.744 \times 10^{-3}$ \\
& 0.9 & $2.955 \times 10^{-3}$ & $2.961 \times 10^{-3}$ \\
10 & 0.5 & $1.594 \times 10^{-2}$ & $1.607 \times 10^{-2}$ \\
& 0.8 & $7.124 \times 10^{-3}$ & $7.142 \times 10^{-3}$ \\
0.9 & $3.673 \times 10^{-3}$ & $3.669 \times 10^{-3}$ \\
\hline \hline
\end{tabular}




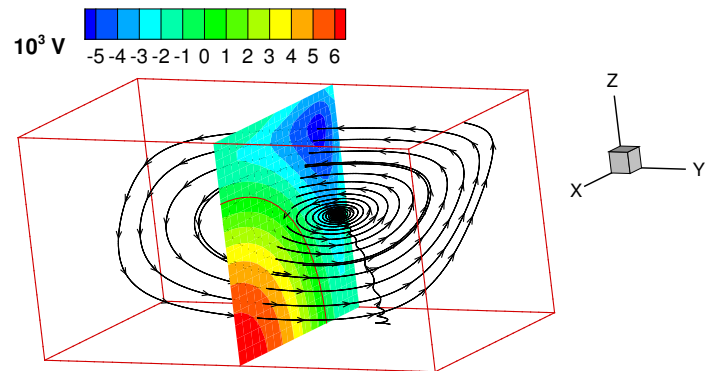

(a) $\mathrm{Kn}=0.1, L_{y} / L_{x}=1$

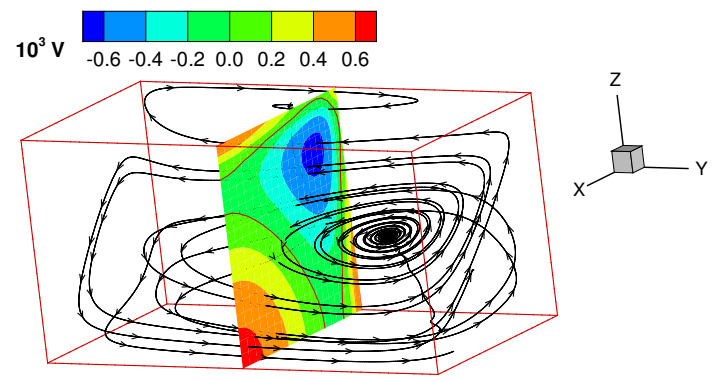

(c) $\mathrm{Kn}=1, L_{y} / L_{x}=1$

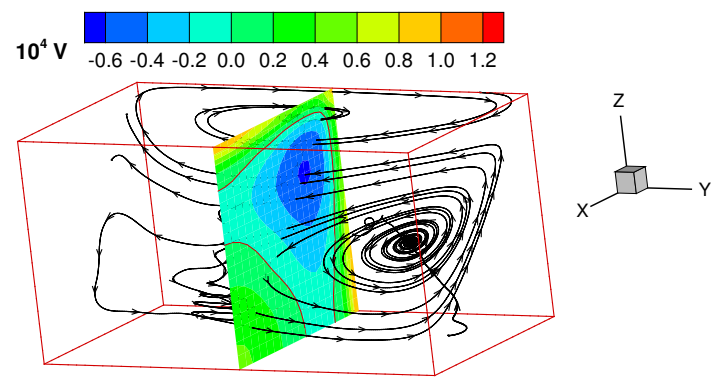

(e) $\mathrm{Kn}=10, L_{y} / L_{x}=1$

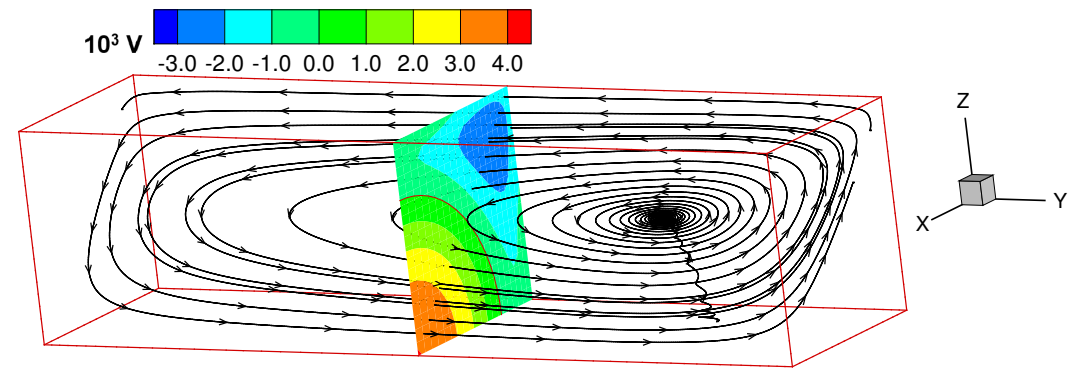

(b) $\mathrm{Kn}=0.1, L_{y} / L_{x}=2$

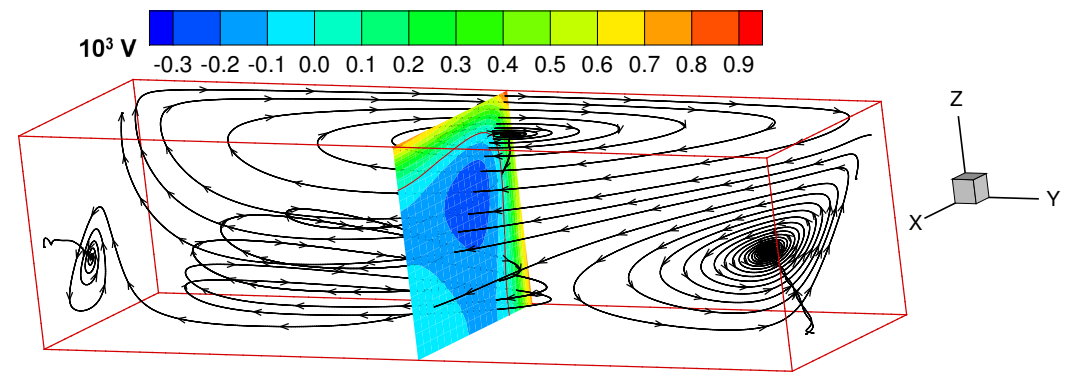

(d) $\mathrm{Kn}=1, L_{y} / L_{x}=2$

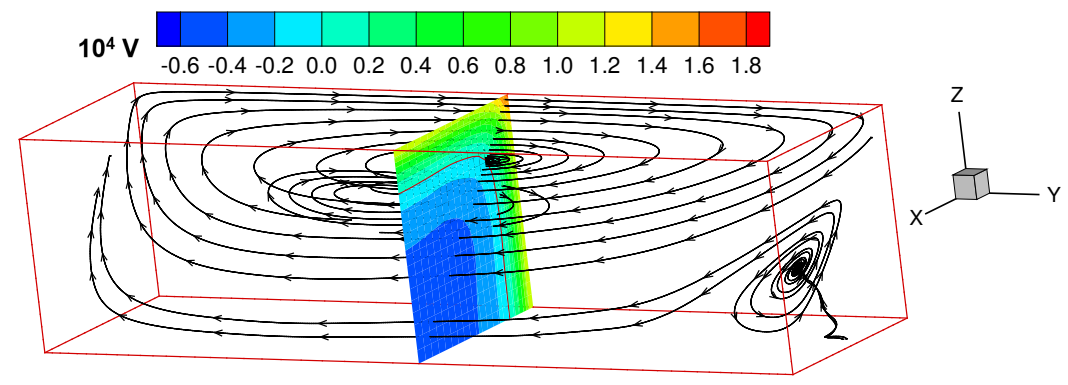

(f) $\mathrm{Kn}=10, L_{y} / L_{x}=2$

FIG. 9. Streamlines and sliced contour plots of V-velocity at different Knudsen numbers and aspect ratios. Only one quarter of the domain $(x \in[0,0.5], z \in[0.5,1])$ is shown due to the two-fold symmetry in $\mathrm{X}$ and $\mathrm{Z}$ directions. In each sub-figure, the contour plot is on the slice at the center position along the Y direction. In each contour plots, the red solid line(s) indicate(s) the contour level of 0 . The streamlines are only shown in the two diagonal planes. The temperature ratio is set as the base configuration, i.e., $T_{c} / T_{h}=0.5$.

are shown in Fig. 9. Comparing the results in Fig. 9 with those in Fig. 2, we can observe that the flow field becomes more complex as $L_{y} / L_{x}$ reduces. For small $L_{y} / L_{x}$, the type-I vortex ring near the cold end occupies a much larger portion of the channel than the cases of $L_{y} / L_{x}=5$. Moreover, for the cases of $L_{y} / L_{x}=1$, the type-I vortex ring dominates the flow field even though Kn increases to 10 [see Fig. 9(e)]. The strong type-I vortex ring in the cases of $L_{y} / L_{x}=1$ leads to an interesting contrast that, for $L_{y} / L_{x}=5$ and 2 , the flow direction along the Y-direction center lines inverts as Kn increases from 0.1 to 10 , while for $L_{y} / L_{x}=1$, such invert never happens. This can be seen more clearly in Fig. 10, where the V-velocity profiles along the Y-direction center lines are shown. Comparing Figs. 10(a), (b) and (c), it can be seen that the $\mathrm{V}$-velocity profiles for the cases of $L_{y} / L_{x}=1$ (blue lines in the figures) are always positive.

To analyze the flow features more clearly, we extract the flow fields on the $\mathrm{Y}-\mathrm{Z}$ symmetric plane of the channel and show the streamlines and isothermals in Fig. 11. More detailed vortex ring structures can be observed from the two-dimensional slices. Figure 11 shows that, for $\mathrm{Kn}=0.1$, the flow is relatively simple and only type-I vortex ring appears. As Kn increase to 1, the type-II vortex ring develops quickly in the cases of $L_{y} / L_{x}=2$ and a new vortex ring 


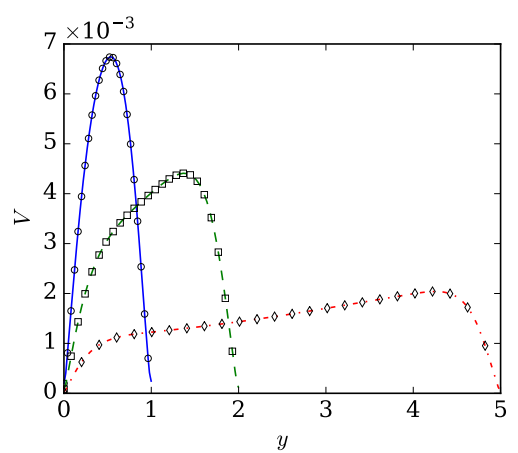

(a)

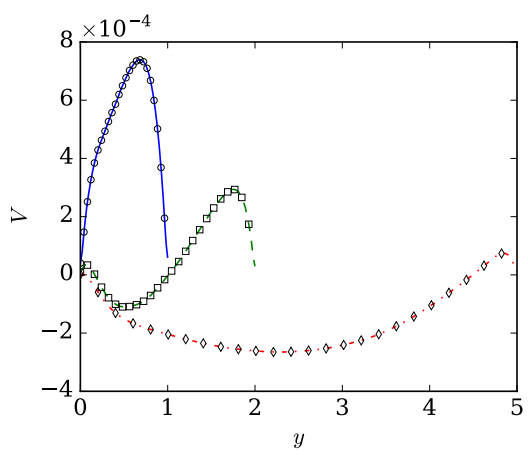

(b)

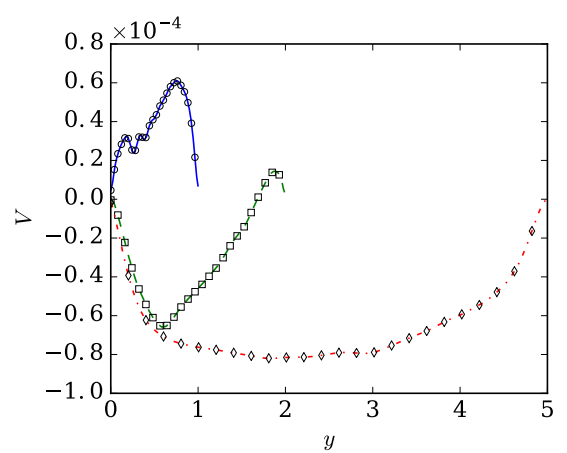

(c)

FIG. 10. V-velocity profiles along the Y-direction center lines of the channel at different aspect ratios $\left(\left(\rightarrow-: L_{y} / L_{x}=1,-\sigma^{-}:\right.\right.$ $\left.L_{y} / L_{x}=2,--_{-}: L_{y} / L_{x}=5\right)$.) and Knudsen numbers. (a) $\mathrm{Kn}=0.1$, (b) $\mathrm{Kn}=1$, (c) $\mathrm{Kn}=10$. The temperature ratio is set as the base configuration, i.e., $T_{c} / T_{h}=0.5$.

is formed near the hot end [see Fig. 11(d)]. The new vortex is not observed as Kn increases further to 10, in which case the type-II vortex ring dominates the flow field [see Fig. 11(f)]. For the cases of $L_{y} / L_{x}=1$, the development of type-II vortex ring is not so quick when Kn increases. This is because the type-II vortex ring develops from the hot end edges to the cold end, but the development is limited soon by the type-I vortex ring due to the limited space along the Y direction. In addition, we can observe a new vortex ring near the hot end in the cases of $L_{y} / L_{x}=1$ as Kn increases to 10, which are not identified in other cases [see Fig. 11(e)].

The average heat flux on the two ends are also measured for each of the aspect ratios and the results are listed in Table IV. Obviously, the heat flux is enhanced due to the enlarged temperature gradient as $L_{y} / L_{x}$ decreases.

TABLE IV. Average heat flux $\bar{q}_{y}$ of the hot (left) and cold (right) ends at various Knudsen numbers and channel aspect ratios.

\begin{tabular}{llll}
\hline \hline Kn $L_{y} / L_{x}$ & Cold side & Hot side \\
\hline 0.1 & 1 & $2.509 \times 10^{-2}$ & $2.477 \times 10^{-2}$ \\
& 2 & $1.349 \times 10^{-2}$ & $1.241 \times 10^{-2}$ \\
& 5 & $5.573 \times 10^{-3}$ & $4.729 \times 10^{-3}$ \\
1 & 1 & $4.966 \times 10^{-2}$ & $5.055 \times 10^{-2}$ \\
& 2 & $2.939 \times 10^{-2}$ & $3.015 \times 10^{-2}$ \\
& 5 & $1.264 \times 10^{-2}$ & $1.280 \times 10^{-2}$ \\
10 & 1 & $5.652 \times 10^{-2}$ & $5.668 \times 10^{-2}$ \\
& 2 & $3.513 \times 10^{-2}$ & $3.532 \times 10^{-2}$ \\
& 5 & $1.594 \times 10^{-2}$ & $1.607 \times 10^{-2}$ \\
\hline \hline
\end{tabular}

\section{CONCLUDING REMARKS}

The thermally induced flow rarefied gas flow in a three-dimensional enclosure with a square cross-section is numerically investigated using a discrete velocity method based on the Shakhov kinetic equation. The Knudsen number dependencies of the vortex rings formed in the enclosure and their developments have been analyzed. Effects of the temperature ratio between the enclosure walls and the aspect ratio have been investigated. The heat transfer behavior has also been studied.

The vortex ring structures vary significantly with the changing of $\mathrm{Kn}$ in the early transition regime. The previously reported invert of thermal transpiration flow direction near the non-isothermal walls in similar but two-dimensional configurations has also been observed in this three-dimensional enclosure. The hot-to-cold slip flow begins near the hot-end edges in the early transition regime and quickly develops into a vortex ring as Kn increases. As a special feature due to the three-dimensionality, the slip flow near the lateral edges is much stronger than near the lateral sides. The temperature ratio and aspect ratio are found to influence the flow field and heat transfer as both of them affect the degree of non-equilibrium of the distribution function. Especially, for a cubic-like enclosure, a new type of 


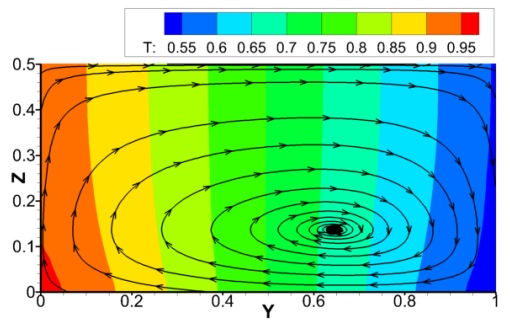

(a) $\mathrm{Kn}=0.1, L_{y} / L_{x}=1$

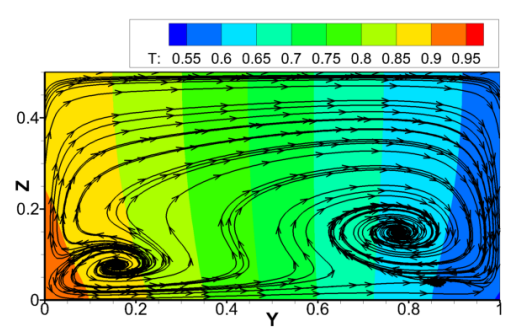

(c) $\mathrm{Kn}=1, L_{y} / L_{x}=1$

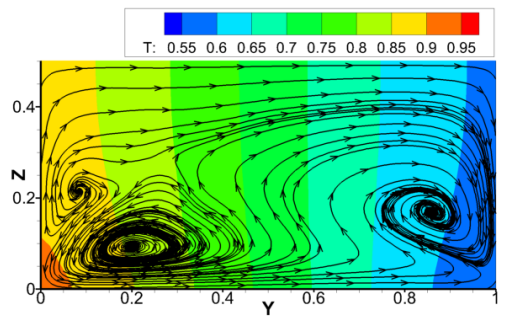

(e) $\mathrm{Kn}=10, L_{y} / L_{x}=1$

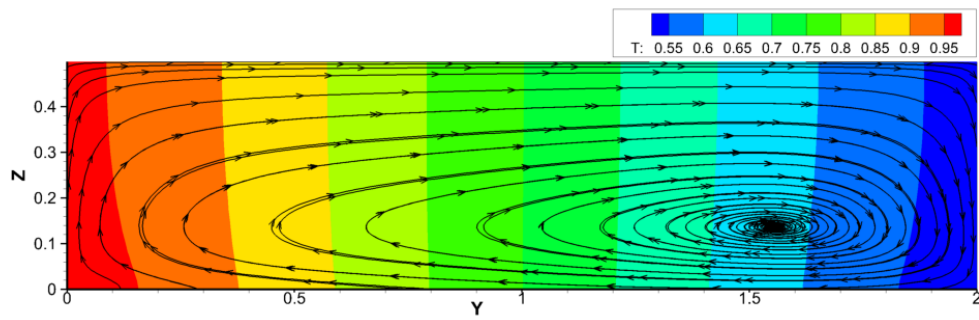

(b) $\mathrm{Kn}=0.1, L_{y} / L_{x}=2$

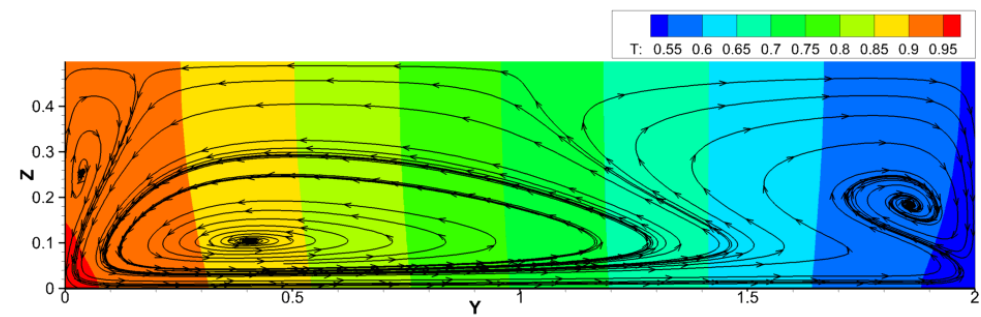

(d) $\mathrm{Kn}=1, L_{y} / L_{x}=2$

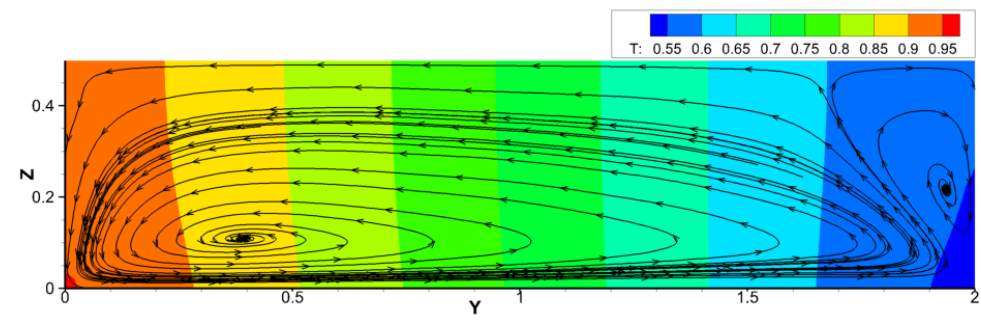

(f) $\mathrm{Kn}=10, L_{y} / L_{x}=2$

FIG. 11. Streamlines and contour plots of temperature on the Y-Z symmetric plane at different Knudsen numbers and aspect ratios. The temperature ratio is set as the base configuration, i.e., $T_{c} / T_{h}=0.5$. Only one half of the symmetric plane $(z \in[0,0.5])$ is shown due to the symmetry in $\mathrm{Z}$ direction.

vortex ring is observed near the hot-end edges at high Knudsen number and is not previously observed in studies of two-dimensional similar configurations.

We believed that the current three-dimensional study of thermally induced flow has scientific interest as the detailed flow structures have been obtained. The cases studied can also serve as reference examples to benchmark novel efficient numerical method for modeling extremely low-speed rarefied gas flow. In addition, the results and analyses are also valuable for guiding practical designs of devices in MEMS and vacuum industries where thermally induced flows are important.

\section{ACKNOWLEDGMENTS}

This research work was financially supported by the National Key Research and Development Plan with grant No. 2016YFB0600805 and the Special program for Applied Research on Super Computation of the NSFC-Guangdong Joint Fund (the second phase). L. Zhu would like to acknowledge Mr. Chuang Zhang and Dr. Songze Chen for helpful discussions on implementing and verification of the iterative scheme, and Dr. Lei Wu's for the suggestion of using the nonuniform velocity grid. The financial support to L. Zhu from Prof. Yonghao Zhang and the Chinese Scholarship Council (CSC) during his visit to the UK (CSC Student No. 201606160050) are also greatly acknowledged. 


\section{Appendix: Grid independence verifications in both physical and velocity spaces}

For the currently adopted deterministic computation of the Shakhov equation, the grid independencies in both the physical and velocity spaces should be verified. As the thermally induced flow is extremely slow compared with the molecular thermal velocity and the discrete velocities, it is very sensitive to the choice of the discrete velocity set and the numerical quadrature rule. The non-smoothness of the distribution function for high Knudsen number flow makes the problem more challenging. On the contrary, as the shear flow near the wall is not strong, i.e., the characteristic Reynolds number is small, the numerical results are not sensitive to the physical space grid. We show here only the verification of results on the velocity grid for the case of $\mathrm{Kn}=10$, and on the physical space grid for the cases of $\mathrm{Kn}=0.01$ and $\mathrm{Kn}=0.1$.

A detailed comparison has been conducted with regard to the choice of numerical quadrature rules and grid resolutions in velocity space. The quadrature rules tested include the compound Newton-Cotes rule, sphere coordinate based Gauss-Legendre rule [43] and the trapezoidal rule with nonuniform grid [see Eq. (12)]. The trapezoidal rule with nonuniform grid gives the best results with the same computing expense when the Knudsen number is high. Thus we adopted this rule for all high Knudsen number cases. The velocities profiles along the geometric center lines of the channel obtained with different numbers of grid points [M in Eq. (12)] are shown in Fig. 12. The two velocity grids used are $64^{3}$ and $128^{3}$ points, respectively. The physical space grid used is the coarser mesh identified by the physical space grid independence study (see below). Figure 12 shows that the two results agree quite well with each other, except that the velocity profile with the $64^{3}$ velocity grid is slightly non-smooth in the center region of the channel. Given such overall acceptable agreements, we use the $64^{3}$ velocity grid for all cases with $\mathrm{Kn} \geq 1$. The velocity grids used for other Knudsen numbers are listed in Table V.

The physical space grids used for all cases are linearly stretched meshes with the cell size varies exponentially from the walls to the channel center. We simulate the cases of $\mathrm{Kn}=0.01$ and 0.1 on two grids with sizes of $40 \times 40 \times 80$ (the coarse grid) and $80 \times 80 \times 120$ (the fine grid). The smallest cell sizes $\left(\Delta x_{\min } \times \Delta y_{\min } \times \Delta z_{\min }\right)$ near the wall are $0.02^{3}$ and $0.01^{3}$, respectively, as listed in Table VI. The V-velocity profiles across the center lines of the channel are presented in Fig. 13, from which we can see the results on the fine and coarse grids match with each other quite well. Thus, we use the coarse grid for all cases with $L_{y} / L_{x}=5$. For cases with other aspect ratios, i.e., $L_{y} / L_{x}=2$ and 1 , we simply change the cell number in the Y direction, i.e., NY in Table VI.

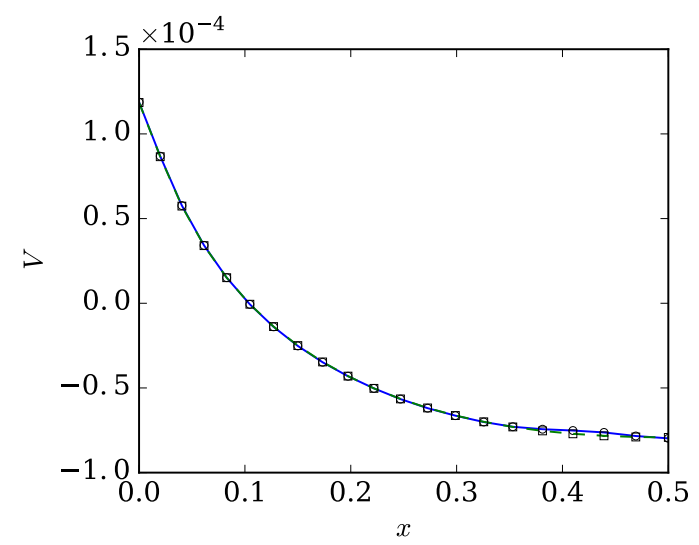

(a)

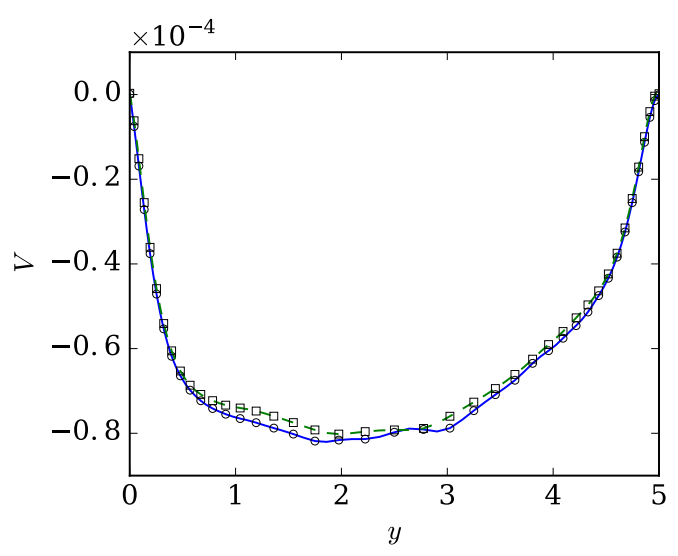

(b)

FIG. 12. V-velocity profiles along the X-(a) and Y-(b) direction center lines obtained on different velocity space grids $(\rightarrow-$ : nonuniform $64^{3}$, : $:$ nonuniform $128^{3}$ ) for the cases of $\mathrm{Kn}=10$. 


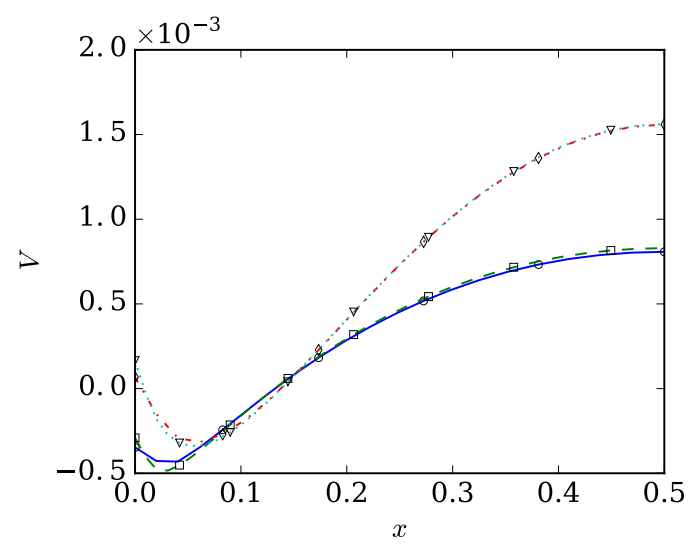

(a)

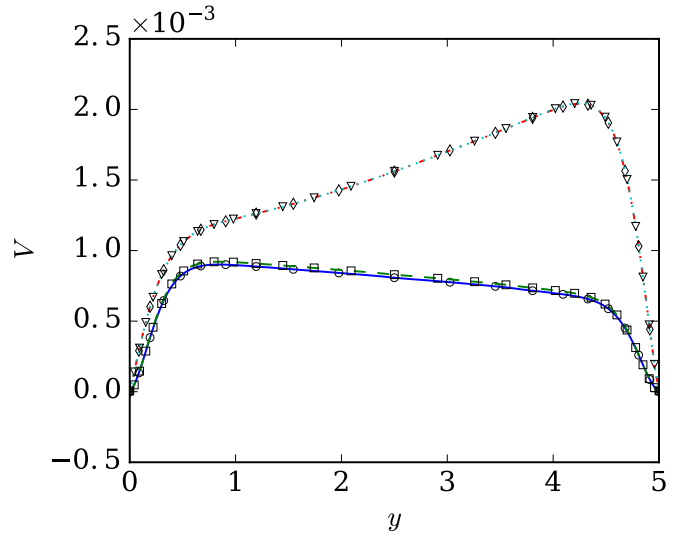

(b)

FIG. 13. V-velocity profiles along the X-(a) and Y-(b) direction center lines obtained on different physical space grids for the cases of $\mathrm{Kn}=0.01$ and $\mathrm{Kn}=0.1 . \rightarrow$ : coarse grid $\mathrm{Kn}=0.01,-\bullet^{-}:$fine grid $\mathrm{Kn}=0.01,--_{-}:$coarse grid $\mathrm{Kn}=0.1,-\nabla^{\prime}:$ fine grid $\mathrm{Kn}=0.1$.

TABLE V. Discrete velocity sets used in the simulations.

\begin{tabular}{lll}
\hline \hline Kn & Discrete velocity set & Velocity grid size \\
\hline 0.01 & Gauss-Hermite & $16 \times 16 \times 16$ \\
0.1 & Gauss-Hermite & $28 \times 28 \times 28$ \\
0.2 & Gauss-Hermite & $28 \times 28 \times 28$ \\
0.5 & Gauss-Hermite & $28 \times 28 \times 28$ \\
1 & Nonuniform & $64 \times 64 \times 64$ \\
10 & Nonuniform & $64 \times 64 \times 64$ \\
\hline \hline
\end{tabular}

TABLE VI. Physical space grids used in the simulations for different channel aspect ratios. The entry with $5^{*}$ represents the finer physical space mesh used in the grid independence study. All grids are linearly stretched grid with the cell size in each direction grows exponentially towards the geometrical center.

\begin{tabular}{lllll}
\hline \hline$L_{y} / L_{x}$ & $\mathrm{NX}(\mathrm{NZ})$ & $\mathrm{NY}$ & $\Delta x_{\min }\left(\Delta z_{\min }\right)$ & $\Delta y_{\min }$ \\
\hline 5 & 40 & 80 & 0.02 & 0.02 \\
$5^{*}$ & 60 & 120 & 0.01 & 0.01 \\
2 & 40 & 50 & 0.02 & 0.02 \\
1 & 40 & 40 & 0.02 & 0.02 \\
\hline \hline
\end{tabular}




\section{REFERENCES}

[1] Y. Sone, Molecular Gas Dynamics: Theory, Techniques, and Applications (Birkhäuser, 2007).

[2] O. Reynolds, "On certain dimensional properties of matter in the gaseous state," Proceedings of the Royal Society of London 28, 303-321 (1878).

[3] J. C. Maxwell, "On stresses in rarified gases arising from inequalities of temperature," Philosophical Transactions of the Royal Society of London 170, 231-256 (1879).

[4] M. Knudsen, "Thermischer molekulardruck der gase in röhren," Annalen der Physik 338, 1435-1448 (1910).

[5] S. McNamara and Y. B. Gianchandani, "On-chip vacuum generated by a micromachined Knudsen pump," Journal of Microelectromechanical Systems 14, 741-746 (2005).

[6] A. Seungdo, N. K. Gupta, and Y. B. Gianchandani, "A Si-micromachined 162-stage two-part Knudsen pump for on-chip vacuum," Journal of Microelectromechanical Systems 23, 406-416 (2014).

[7] H. Yamaguchi, M. Rojas-Cárdenas, P. Perrier, I. Graur, and T. Niimi, "Thermal transpiration flow through a single rectangular channel," Journal of Fluid Mechanics 744, 169-182 (2014).

[8] M. Cardenas, I. Graur, P. Perrier, and J. G. Meolans, "Thermal transpiration flow: A circular cross-section microtube submitted to a temperature gradient," Physics of Fluids 23, 031702 (2011).

[9] Y. L. Han, E. P. Muntz, A. Alexeenko, and M. Young, "Experimental and computational studies of temperature gradientdriven molecular transport in gas flows through nano/microscale channels," Nanoscale and Microscale Thermophysical Engineering 11, 151-175 (2007).

[10] S. K. Loyalka, T. S. Storvick, and H. S. Park, "Poiseuille flow and thermal creep flow in long, rectangular channels in molecular and transition-flow regimes," Journal of Vacuum Science \& Technology 13, 1188-1192 (1976).

[11] T. Ohwada, Y. Sone, and K. Aoki, "Numerical analysis of the Poiseuille and thermal transpiration flows between two parallel plates on the basis of the Boltzmann equation for hard-sphere molecules," Physics of Fluids A 1, 2042-2049 (1989).

[12] F. Sharipov and V. Seleznev, "Data on internal rarefied gas flows," Journal of Physical and Chemical Reference Data 27, 657-706 (1998).

[13] I. Graur and F. Sharipov, "Non-isothermal flow of rarefied gas through a long pipe with elliptic cross section," Microfluidics and Nanofluidics 6, 267-275 (2009).

[14] V. A. Titarev, "Implicit high-order method for calculating rarefied gas flow in a planar microchannel," Journal of Computational Physics 231, 109-134 (2012).

[15] H. Liu, M. Wang, J. Wang, G Zhang, H. Liao, R. Huang, and X. Zhang, "Monte Carlo simulations of gas flow and heat transfer in vacuum packaged MEMS devices," Applied Thermal Engineering 27, 323-329 (2007).

[16] A. Ketsdever, N. Gimelshein, S. Gimelshein, and N. Selden, "Radiometric phenomena: From the 19th to the 21st century," Vacuum 86, 1644-1662 (2012).

[17] D. H. Papadopoulos and D. E. Rosner, "Enclosure gas flows driven by non-isothermal walls," Physics of Fluids 7, 2535-2537 (1995).

[18] R. Anirudh, T. Manuel, and S. Henning, "Heat transfer in micro devices packaged in partial vacuum," Journal of Physics: Conference Series 362, 012034 (2012).

[19] J.-C. Huang, K. Xu, and P. Yu, "A unified gas-kinetic scheme for continuum and rarefied flows III: Microflow simulations," Communications in Computational Physics 14, 1147-1173 (2013).

[20] M. Vargas, G. Tatsios, D. Valougeorgis, and S. Stefanov, "Rarefied gas flow in a rectangular enclosure induced by nonisothermal walls," Physics of Fluids 26, 057101 (2014).

[21] L. Wu, J. M. Reese, and Y. Zhang, "Solving the Boltzmann equation deterministically by the fast spectral method: application to gas microflows," Journal of Fluid Mechanics 746, 53-84 (2014).

[22] J. Chen, L. Baldas, and S. Colin, "Numerical study of thermal creep flow between two ratchet surfaces," Vacuum 109, 294-301 (2014).

[23] G. A. Bird, Molecular Gas Dynamics and the Direct Simulation of Gas Flows (Clarendon Press, 1994).

[24] N. D. Masters and W. Ye, "Octant flux splitting information preservation DSMC method for thermally driven flows," Journal of Computational Physics 226, 2044-2062 (2007).

[25] G. A. Radtke, N. G. Hadjiconstantinou, and W. Wagner, "Low-noise Monte Carlo simulation of the variable hard sphere gas," Physics of Fluids 23, 030606 (2011).

[26] A. Mohammadzadeh, A. S. Rana, and H. Struchtrup, "Thermal stress vs. thermal transpiration: A competition in thermally driven cavity flows," Physics of Fluids 27, 112001 (2015).

[27] L. Zhu, S. Chen, and Z. Guo, "dugksFoam: An open source OpenFOAM solver for the Boltzmann model equation," Computer Physics Communications 213, 155-164 (2017).

[28] L. Wu, C. White, T. J. Scanlon, J. M. Reese, and Y. Zhang, "Deterministic numerical solutions of the boltzmann equation using the fast spectral method," Journal of Computational Physics 250, 27-52 (2013).

[29] E. M. Shakhov, "Generalization of the Krook kinetic relaxation equation," Fluid Dynamics 3, 95-96 (1968).

[30] S. Chen, C. Zhang, L. Zhu, and Z. Guo, "A unified implicit scheme for kinetic model equations. part I. Memory reduction technique," Science Bulletin 62, 119-129 (2017). 
[31] G. A. Bird, The DSMC Method (CreateSpace Independent Publishing Platform, 2013).

[32] F. Sharipov and G. Bertoldo, "Poiseuille flow and thermal creep based on the Boltzmann equation with the Lennard-Jones potential over a wide range of the Knudsen number," Physics of Fluids 21, 067101 (2009).

[33] S. Chen, K. Xu, and Q. Cai, "A comparison and unification of ellipsoidal statistical and shakhov bgk models," Advances in Applied Mathematics and Mechanics 7, 245-266 (2015).

[34] L. Zhu and Z. Guo, "Application of discrete unified gas kinetic scheme to thermally induced nonequilibrium flows," Computers \& Fluids (2017), 10.1016/j.compfluid.2017.09.019.

[35] F. Sharipov and J. L. Strapasson, "Benchmark problems for mixtures of rarefied gases. I. Couette flow," Physics of Fluids 25, 027101 (2013).

[36] E. Vogel, B. Jäger, R. Hellmann, and E. Bich, "Ab initio pair potential energy curve for the argon atom pair and thermophysical properties for the dilute argon gas. II. Thermophysical properties for low-density argon," Molecular Physics 108, 3335-3352 (2010).

[37] F. Sharipov, Rarefied gas dynamics: fundamentals for research and practice (John Wiley \& Sons, 2015).

[38] W. Su, S. Lindsay, H. Liu, and L. Wu, "Comparative study of the discrete velocity and lattice Boltzmann methods for rarefied gas flows through irregular channels," Physical Review E 96, 023309 (2017).

[39] K. Xu and J.-C. Huang, "A unified gas-kinetic scheme for continuum and rarefied flows," Journal of Computational Physics 229, 7747-7764 (2010).

[40] Z. Guo, R. Wang, and K. Xu, "Discrete unified gas kinetic scheme for all Knudsen number flows. II. thermal compressible case," Physical Review E 91, 033313 (2015).

[41] Y. Zhu, C. Zhong, and K. Xu, "Implicit unified gas-kinetic scheme for steady state solutions in all flow regimes," Journal of Computational Physics 315, 16-38 (2016).

[42] Y. Zhu, C. Zhong, and K. Xu, "Unified gas-kinetic scheme with multigrid convergence for rarefied flow study," Physics of Fluids 29, 096102 (2017).

[43] V. E. Ambruş and V. Sofonea, "High-order thermal lattice Boltzmann models derived by means of Gauss quadrature in the spherical coordinate system," Physics Review E 86, 016708 (2012). 